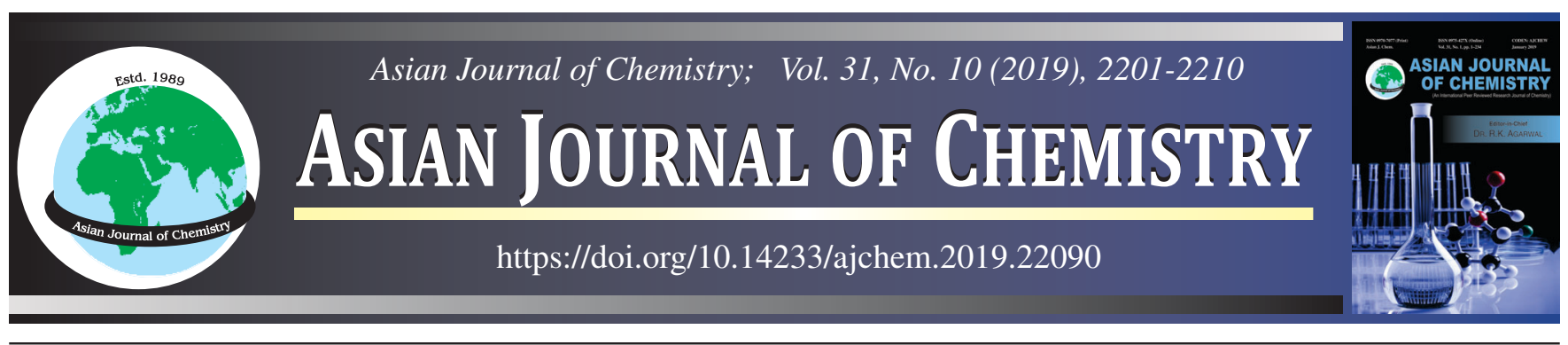

\title{
Synthesis and Antimicrobial Activity of Dithiocarbamates of w-Substituted (2-naphthyloxy)alkanes
}

\section{Sadaf Zaidi ${ }^{1, *}$, Devdutt Chaturvedi ${ }^{2}$, Mridula Saxena $^{1}$ and Richa Srivastava ${ }^{3}$}

${ }^{1}$ Department of Applied Chemistry, Amity School of Applied Sciences, Amity University Uttar Pradesh, Lucknow Campus, Lucknow-226028, India ${ }^{2}$ Department of Chemistry, School of Physical \& Material Sciences, Mahatma Gandhi Central University, Motihari-845401, India

${ }^{3}$ Amity Institute of Pharmacy, Amity University Uttar Pradesh, Lucknow Campus, Lucknow-226028, India

*Corresponding author: E-mail: sadaf.zaidi00@gmail.com

A series of dithiocarbamates of $\omega$-substituted (2-naphthyloxy) alkanes was developed through condensation of 2-(2-chloro-alkoxy)naphthalene to various kinds of aliphatic, aromatic, alicyclic, heterocyclic primary and secondary amines employing benzyl trimethyl ammonium hydroxide in catalytic quantity (Triton-B/CS 2 system) afforded desired products in high yields (82-98 \%). The complete series of synthesized compounds (4-48) were evaluated for antimicrobial activity through microdilution method using various bacterial and fungal strains. The antifungal and antibacterial values were estimated as MIC values. Fluconazole and ciprofloxacin [16 to $0.03 \mu \mathrm{g} /$ $\mathrm{mL}$ ] were used as the standard antifungal and antibacterial drug, respectively. Out of series of evaluated compounds, some of these compounds such as compounds $\mathbf{2 8}, \mathbf{2 9}, \mathbf{3 0}, \mathbf{3 1}, \mathbf{3 2}, \mathbf{3 3}$ have displayed maximum potency which is comparable to standard drugs.

Keywords: Dithiocarbamates, Antimicrobial, Amines.

ᄂ

\section{INTRODUCTION}

Dithiocarbamates have always received the attention of the researchers round the world because of its wide utility in areas such as pharmaceuticals [1-4], intermediate product in organic synthesis [5], for the sheilding of amino groups in peptide chemistry $[6,7]$ and as linking agents in combinatorial chemistry [8-10]. Organic dithiocarbamates have been extensively used as intermediate for the synthesis of structurally diverse synthetic intermediates/molecules of biological significance like antimalarial [11], anticholinergic [12], antimicrobial [13], antimitotic [14], antitubercular [15], antifungal [16], anticancer [17], antioxidant [18], antiprotozoal [19], antileprosy [20], antifolates [21], antitubulin [22], antialzheimer [23], anti-HIV [24], antipoliferative [25] and anticontraceptives [26] active agents. As a useful synthon organic dithiocarbamates have been extensively used for the synthesis of structurally diverse biological potent synthetic intermediates/molecules like isothiocyanates [27], thiourea [28], cynamide [29], dithiobenzophene [30], glycosides [31], $\beta$-sulphonamides [32], amide [33], dicarboxylates [34], thiadizoles [35], dithiolanes [36], thiones [37], benzimidazole [38], carbamate [39], pyran [40] and flavonoids [41], etc. Apart from above mentioned activities, dithiocarba-mates of various imidazole [42], brassinin [43], rhodanine [44], quinoline [45], metal complexes [46], ammonium salts [47], etc. derivative have emerged as potent antimicrobial agents. As our group is working in drug discovery through design and synthesis of novel class of natural/semi-synthetic/ synthetic molecules especially molecules like carbamates, dithiocarbamates, dithiocarbazates, etc. Keeping in view the importance of dithiocarbamates and its derivatives, we became interested in investigating various structurally diverse biologically potent compounds.

Considering the potency of dithiocarbamates as antimicrobial agents, we became interested to investigate the antimicrobial activity of dithiocarbamates of $\omega$-substituted (2-naphthyloxy) alkanes (Prototype I).

\section{EXPERIMENTAL}

Procedure for $\omega$-substituted 2-naphthyloxy haloalkanes (3): Measured amount of $\beta$-naphthol (1) was taken in dry acetone

This is an open access journal, and articles are distributed under the terms of the Attribution 4.0 International (CC BY 4.0) License. This license lets others distribute, remix, tweak, and build upon your work, even commercially, as long as they credit the author for the original creation. You must give appropriate credit, provide a link to the license, and indicate if changes were made. 
<smiles>S=C(SCCOc1ccc2ccccc2c1)N1[B]CCCC1</smiles>

Prototype I $(\mathrm{n}=1,2 \& 3$; Compounds 4-48)

and anhyd. $\mathrm{K}_{2} \mathrm{CO}_{3}$ (10 eqv.) was added in it. To this, 1-bromo3 -chloro propane (2) was added ( 2.5 eqv.) and then the reaction was refluxed for $12-15 \mathrm{~h}$. The continuous monitoring of progress of reaction was done by TLC which indicates the appearance of less polar new spot. The filtrate of reaction mixture was concentrated, extracted thrice with ethyl acetate. After separation the organic layer was dried over anhydrous $\mathrm{Na}_{2} \mathrm{SO}_{4}$ afforded the corresponding compound $\mathbf{3}$. Compound $\mathbf{3}$ was confirmed by various spectroscopic and analytical techniques.

Procedure for synthesis of dithiocarbamates of Prototype I (4-48): Measured amount of desired amine was dissolved in dry DMSO. To this, measured amount of $\mathrm{CS}_{2}$ and Triton-B were added drop by drop and the reaction was allowed to stir for $15 \mathrm{~min}$. After adding compound $\mathbf{3}$ to the reaction mixture and the reaction was stirred for about 20-40 min. Monitoring of reaction progress was done by TLC. Triple extraction of reaction mixture was done with ethyl acetate once the reaction was complete. The organic layer was separated, dried over anhydrous $\mathrm{Na}_{2} \mathrm{SO}_{4}$ afforded the final product that is, dithiocarbamates of prototype I (compounds 4-48).

Biological activities: The antibacterial and antifungal activities of the $\omega$-substituted (2-naphthyloxy) alkanes against bacterial strains (Staphylococcus aureus ATCC 29313, Methicillin resistant Staphylococcus aureus), two Gram-negative strains (Escherichia coli ATCC 25922, Pseudomonas aeruginosa ATCC 27853), two yeast strains (Candida albicans ATCC 22019, S. schencki) and two filamentous fungi (Aspergillus fumigatus LSI-II, Aspergillus niger ATCC 16404) was performed by using microdilution method. Antibacterial tests were performed using Muller Hinton Broth which was buffered to $\mathrm{pH}$ 7.0. The antifungal testing was performed using RPMI 1640 with L-glutamine buffered to $\mathrm{pH}$ 7.0. It was supplemented with $0.165 \mathrm{M} 3-(\mathrm{N}$-morpholino) propanesulfonic acid (MOPS) [Sigma-Aldrich]. The stock solution of the compounds was prepared using DMSO. The minimum inhibitory concentration (MIC) of the compounds was determined by serial 2 fold diluting the oils in the abovementioned media in $100 \mathrm{~mL}$ volume in a 96 well $\mathrm{U}$ bottom microtitre plate. The final concentrations of compound ranged from 128 to $0.25 \mu \mathrm{g} / \mathrm{mL}$. Flucanazone and ciprofloxacin [16 to $0.03 \mu \mathrm{g} / \mathrm{mL}$ ] (Sigma-Aldrich) were used as standard antifungal and antibacterial agents respectively. The bacterial and fungal suspension of the overnight grown bacterial and fungal was prepared in sterile normal saline and their density was adjusted to $0.5 \mathrm{McF}$ arland. The bacterial cultures were diluted and added in $100 \mathrm{~mL}$ volume to a final inoculum of $1 \times 10^{5} \mathrm{CFU} / \mathrm{mL}$. For fungal cultures $1 \times 10^{3} \mathrm{CFU} / \mathrm{mL}$ was used. The plates were incubated at $37{ }^{\circ} \mathrm{C}$ for $24 \mathrm{~h}$ for bacterial cultures and $48 \mathrm{~h}$ for fungal cultures. The plates were read visually and the minimum concentration of the compounds showing no turbidity was recorded as MIC.

All the chemicals used were obtained from Merck, Aldrich and Fluka chemical companies. Reactions were carried out in nitrogenous atmosphere. The structural analysis of compound was done as IR spectra (4000-200 $\mathrm{cm}^{-1}$ ) on Bomem MB-104FTIR spectrophotometer where as NMRs were scanned on AC-300F, NMR ( $300 \mathrm{MHz}$ ), instrument using $\mathrm{CDCl}_{3}$ and some other deutrated solvents and TMS as internal standard. Elemental analysis were made by Carlo-Erba EA 1110-CNNO$\mathrm{S}$ analyzer and the obtained values were in accordance with calculated values.

General procedure for the preparation of $\omega$-naphthyloxy halo alkanes (3a-c) involves refluxing of mixture of $\beta$-naphthol (1) (20 g, $0.14 \mathrm{~mol})$, anhydrous $\mathrm{K}_{2} \mathrm{CO}_{3}(100 \mathrm{~g}$, in excess) and bromochloroalkane $2(0.14 \mathrm{~mol})$ in dry acetone $(200 \mathrm{~mL})$ for $12-15 \mathrm{~h}$. Reaction mixture was filtered and filtrate was concentrated to get oily compound, which was crystallized with benzene-hexane to give the colourless crystals of pure desired compound (Scheme-I) [48].

2-(2-Naphthyloxy)-1-chloroethane (3a): Yield: $27.4 \mathrm{~g}$ (96 \%); m.p.: $94{ }^{\circ} \mathrm{C}$; IR (KBr, $\left.v_{\max }, \mathrm{cm}^{-1}\right): 1455$ (Ar), 1507 (Ar), 1586 (Ar), $2878(\mathrm{CH}), 2927(\mathrm{CH}) ;{ }^{1} \mathrm{H}$ NMR (400 MHz, $\left.\mathrm{CDCl}_{3}\right): \delta=3.82\left(\mathrm{t}, 2 \mathrm{H}, \mathrm{CH}_{2} \mathrm{Cl}\right), 4.24\left(\mathrm{t}, 2 \mathrm{H}, \mathrm{OCH}_{2}\right), 6.97-$ $7.65(\mathrm{~m}, 7 \mathrm{H}, \mathrm{Ar}-\mathrm{H}) ;{ }^{13} \mathrm{C} \mathrm{NMR}\left(100 \mathrm{MHz}, \mathrm{CDCl}_{3}\right): \delta=45.2$, $75.2,105.7,118.5,123.7,126.5,129.6,134.4,157.6 \mathrm{ppm}$; Mass (EIMS): $m / z=206$; Analysis: $\mathrm{C}_{12} \mathrm{H}_{11} \mathrm{OCl}$, Calcd. (\%): $\mathrm{C}$, 69.74; H, 5.36; Obsd. (\%): C, 70.04, H, 5.66.

3-(2-Naphthyloxy)-1-chloropropane (3b): Yield: 29.6 g $(97 \%)$; m.p.: $98^{\circ} \mathrm{C}$; IR (KBr, $\left.v_{\max }, \mathrm{cm}^{-1}\right): 1462$ (Ar), 1511 (Ar), 1595 (Ar), $2856(\mathrm{CH}), 2942(\mathrm{CH}) ;{ }^{1} \mathrm{H}$ NMR (400 MHz, $\left.\mathrm{CDCl}_{3}\right): \delta=2.26-2.34\left(\mathrm{~m}, 2 \mathrm{H}, \mathrm{CH}_{2}\right), 3.84\left(\mathrm{t}, 2 \mathrm{H}, \mathrm{CH}_{2} \mathrm{Cl}\right)$, $4.24\left(\mathrm{t}, 2 \mathrm{H}, \mathrm{OCH}_{2}\right), 7.12-7.75(\mathrm{~m}, 7 \mathrm{H}, \mathrm{Ar}-\mathrm{H}) \mathrm{ppm}$; Mass (EIMS): $m / z=220$; Analysis: $\mathrm{C}_{13} \mathrm{H}_{13} \mathrm{OCl}$, Calcd. (\%): C, 70.75; H, 5.94; Obsd. (\%): C, 70.79; H, 6.21.

4-(2-Naphthyloxy)-1-chlorobutane (3c): Yield: $34 \mathrm{~g}$ (98 \%); m.p.: $112{ }^{\circ} \mathrm{C}$; IR (KBr, $\left.v_{\max }, \mathrm{cm}^{-1}\right)$ : $1463(\mathrm{Ar}), 1512(\mathrm{Ar})$, 1598 (Ar), $2886(\mathrm{CH}), 2942(\mathrm{CH}) ;{ }^{1} \mathrm{H}$ NMR $(400 \mathrm{MHz}$, $\left.\mathrm{CDCl}_{3}\right): \delta=2.15-2.22\left(\mathrm{~m}, 4 \mathrm{H}, \mathrm{CH}_{2} \mathrm{CH}_{2}\right), 3.77\left(\mathrm{t}, 2 \mathrm{H}, \mathrm{CH}_{2} \mathrm{Cl}\right)$, $4.25\left(\mathrm{t}, 2 \mathrm{H}, \mathrm{OCH}_{2}\right), 7.13-7.76(\mathrm{~m}, 7 \mathrm{H}, \mathrm{Ar}-\mathrm{H}) \mathrm{ppm}$; Mass (EIMS): $m / z=234$; Analysis: $\mathrm{C}_{14} \mathrm{H}_{15} \mathrm{OCl}$, Calcd. (\%): C, 70.72; H, 5.92; Obsd. (\%): C, 70.78; H, 6.20.

Procedure for the preparation of dithiocarbamates of (-substituted (2-naphthyloxy) alkanes: A mixture of desired

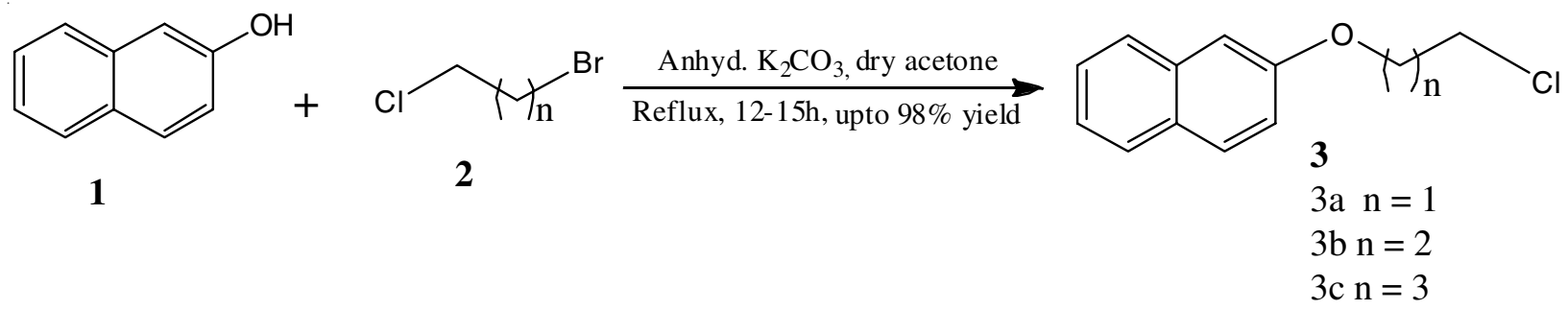


amine $(0.6 \mathrm{~mL}, 5 \mathrm{mmol})$ and carbon disulphide solution $(3 \mathrm{~mL}$, in excess) was taken in dry DMSO $(35 \mathrm{~mL})$. Triton-B $(0.9 \mathrm{~mL}$, $4 \mathrm{mmol}$ ) was added in it and the reaction mixture was stirred at room temperature for $1 \mathrm{~h}$. Now 2-(2-naphthyloxy)-1-chloroalkane $(0.5 \mathrm{~g}, 2 \mathrm{mmol})$ was added in it and reaction was continued till its completion $(2 \mathrm{~h})$ as checked by TLC. Reaction mixture was poured into distilled water $(50 \mathrm{~mL})$ and three-time extraction was done with ethyl acetate. After separation of organic layer, it was dried over anhydrous sodium sulphate and then concentrated to get dithiocarbamate of $\omega$-substituted (2-naphthyloxy) alkanes. This compound was obtained as yellow solid (Scheme-II).

Butyl-dithiocarbamic acid-2-(naphthalen-2-yloxy)ethyl ester (4): Yield: $0.73 \mathrm{~g}(93.5 \%)$; m.p.: $106{ }^{\circ} \mathrm{C}$; IR (KBr, $v_{\max }$, $\mathrm{cm}^{-1}$ ): 661 (C-S), 1114 (C=S), 1454 (Ar), 1511 (Ar), 1612 (Ar), $2864(\mathrm{CH}), 2936(\mathrm{CH}), 3390(\mathrm{NH}) ;{ }^{1} \mathrm{H} \mathrm{NMR}\left(\mathrm{CDCl}_{3}\right): \delta$ $=0.92-0.96\left(\mathrm{t}, 3 \mathrm{H}, \mathrm{CH}_{3}\right), 1.30-1.34\left(\mathrm{~m}, 2 \mathrm{H}, \mathrm{CH}_{2} \mathrm{CH}_{3}\right), 1.53-$ 1.56 (m, 2H, $\mathrm{CH}_{2} . \mathrm{CH}_{2} . \mathrm{CH}_{3}$ ), 2.0 (bs, H, NH), 2.62-2.64 (m, $2 \mathrm{H}, \mathrm{NHCH}_{2}$ ), 3.28-3.32 (t, $\left.2 \mathrm{H}, \mathrm{CH}_{2}-\mathrm{S}-\mathrm{C}=\mathrm{S}\right), 4.71-4.74$ (t, $2 \mathrm{H}$, $\mathrm{CH}_{2}$-O-naphthyl), 6.97-7.64 (m, 7H, Ar-H of naphthyloxy); Mass: m/e 319; Analysis: $\mathrm{C}_{17} \mathrm{H}_{21} \mathrm{NOS}_{2}$, Calcd. (\%): C, 63.91, H 6.63, N, 4.38; Obsd. (\%): C, 64.19, H, 6.49, N, 4.24.

Butyl-dithiocarbamic acid-3-(naphthalene-2-yloxy)propyl ester (5): Yield: 0.73 g (93.5 \%); m.p.: $106{ }^{\circ} \mathrm{C}$; IR $\left(\mathrm{KBr}, \mathrm{v}_{\max }, \mathrm{cm}^{-1}\right): 661(\mathrm{C}-\mathrm{S}), 1115(\mathrm{C}=\mathrm{S}), 1454$ (Ar), 1511 (Ar), 1610 (Ar), 2864 (CH), 2935 (CH), 3390 (NH); ${ }^{1} \mathrm{H}$ NMR $\left(\mathrm{CDCl}_{3}\right): \delta=0.93-0.96\left(\mathrm{t}, 3 \mathrm{H}, \mathrm{CH}_{3}\right), 1.33-1.35(\mathrm{~m}, 2 \mathrm{H}$, $\mathrm{CH}_{2} \mathrm{CH}_{3}$ ), 1.53-1.55 (m, 2H, $\left.\mathrm{CH}_{2} . \mathrm{CH}_{2} . \mathrm{CH}_{3}\right), 2.0$ (bs, H, NH), 2.62-2.65 (m, 2H, $\mathrm{NHCH}_{2}$ ), 2.84-2.86 (t, 2H, $\left.\mathrm{CH}_{2}-\mathrm{S}-\mathrm{C}=\mathrm{S}\right)$, 2.35-2.39 (m, 2H, $\left.\mathrm{CH}_{2} \mathrm{CH}_{2} \mathrm{CH}_{2}\right)$ 4.01-4.05 (t, 2H, $\mathrm{CH}_{2}-\mathrm{O}-$ naphthyl), 6.96-7.62 (m, 7H, Ar-H of naphthyloxy); Mass: m/e 333; Analysis: $\mathrm{C}_{18} \mathrm{H}_{23} \mathrm{NOS}_{2}$, Calcd. (\%): C, 65.91, H 6.83, N, 4.38, S, 19.15, Obsd. (\%): C, 65.82, H, 6.95, N, 4.20, S, $19.23 \%$ : O, 4.80 .

Butyl-dithiocarbamic acid-4-(naphthalene-2-yloxy)butyl ester (6): Yield: $0.73 \mathrm{~g}(93.5 \%)$; m.p.: $106^{\circ} \mathrm{C}$; IR (KBr, $\left.\mathrm{V}_{\max }, \mathrm{cm}^{-1}\right)$ : $661(\mathrm{C}-\mathrm{S}), 1114(\mathrm{C}=\mathrm{S}), 1454(\mathrm{Ar}), 1511$ (Ar), 1612 (Ar), $2864(\mathrm{CH}), 2936(\mathrm{CH}), 3390(\mathrm{NH}) ;{ }^{1} \mathrm{H} \mathrm{NMR}\left(\mathrm{CDCl}_{3}\right): \delta$ $=0.93-0.96\left(\mathrm{t}, 3 \mathrm{H}, \mathrm{CH}_{3}\right), 1.33-1.35\left(\mathrm{~m}, 2 \mathrm{H}, \mathrm{CH}_{2} \mathrm{CH}_{3}\right), 1.53-$ 1.55 (m, 2H, $\mathrm{CH}_{2} . \mathrm{CH}_{2} . \mathrm{CH}_{3}$ ), 2.0 (bs, H, NH), 2.62-2.66 (m, $2 \mathrm{H}, \mathrm{NHCH}_{2}$ ), 2.85-2.87 (t, 2H, $\left.\mathrm{CH}_{2}-\mathrm{S}-\mathrm{C}=\mathrm{S}\right), 1.92-1.96(\mathrm{~m}$, $2 \mathrm{H}, \mathrm{CH}_{2} \mathrm{CH}_{2} \mathrm{CH}_{2}$ ), 1.68-1.71 (m, 2H, $\left.\mathrm{CH}_{2} \mathrm{CH}_{2} \mathrm{CH}_{2}\right), 4.00-4.03$ $\left(\mathrm{t}, 2 \mathrm{H}, \mathrm{CH}_{2}-\mathrm{O}-\right.$ naphthyl), 6.96-7.62 (m, 7H, Ar-H of naphthyloxy); Mass: $m / e$ 347.14; Analysis: $\mathrm{C}_{19} \mathrm{H}_{25} \mathrm{NOS}_{2}$, Calcd. (\%): C, 65.60, H 7.22, N, 4.58; S, 18.42\%: O, 4.58. Obsd. (\%): C, 65.66, H, 7.25, N, 4.60, S, $18.45 \%$ : O, 4.60 .

Hexyl-dithiocarbamic acid-2-(naphthalen-2-yloxy)ethyl ester (7): Yield: $0.8 \mathrm{~g}(96.4 \%)$; m.p.: $119^{\circ} \mathrm{C}$; IR (KBr, $\mathrm{v}_{\max }$, $\mathrm{cm}^{-1}$ ): 665 (C-S), 1116 (C=S), 1475 (Ar), 1514 (Ar), 1602
(Ar), 2875 (CH), 2936 (CH), $3394(\mathrm{NH}) ;{ }^{1} \mathrm{H} \mathrm{NMR}\left(\mathrm{CDCl}_{3}\right): \delta$ $=0.92-0.95\left(\mathrm{t}, 3 \mathrm{H}, \mathrm{CH}_{3}\right), 1.27-1.29\left(\mathrm{~m}, 4 \mathrm{H}, \mathrm{CH}_{2} \mathrm{CH}_{2} \mathrm{CH}_{2} \mathrm{CH}_{3}\right.$ of hexyl group), 1.31-1.35 (m, $2 \mathrm{H}, \mathrm{CH}_{2} \mathrm{CH}_{3}$ of hexyl group), 1.52-1.56 (m, 2H, $\mathrm{CH}_{2} . \mathrm{CH}_{2} . \mathrm{CH}_{3}$ of hexyl group), 2.0 (bs, $\mathrm{H}$, $\mathrm{NH}$ ), 2.36-2.40 (m, 2H, naphthyl-O- $\left.\mathrm{CH}_{2} \mathrm{CH}_{2} \mathrm{CH}_{2}-\mathrm{S}-\mathrm{C}=\mathrm{S}\right)$, 2.63-2.65 (m, 2H, $\mathrm{NHCH}_{2}$ ), 3.24-3.29 (t, 2H, $\left.\mathrm{CH}_{2}-\mathrm{S}-\mathrm{C}=\mathrm{S}\right)$, 4.68-4.73 (t, 2H, $\mathrm{CH}_{2}-\mathrm{O}$-naphthyl), 6.96-7.62 (m, 7H, Ar-H of naphthyloxy); Mass: m/e 347.14; Analysis: $\mathrm{C}_{19} \mathrm{H}_{25} \mathrm{NOS}_{2}$, Calcd. (\%): C, 66.44, H 7.53, N, 3.87, 18.45 \%: O, 4.60. Obsd. (\%): C, 65.66, H, 7.25, N, 4.03, S, $18.45 \%$ : O, 4.60.

Hexyl-dithiocarbamic acid-3-(naphthalene-2-yloxy)propyl ester (8): Yield: $0.8 \mathrm{~g}(96.4 \%)$; m.p.: $119^{\circ} \mathrm{C}$; IR (KBr, $\left.V_{\max }, \mathrm{cm}^{-1}\right)$ : $662(\mathrm{C}-\mathrm{S}), 1116(\mathrm{C}=\mathrm{S}), 1475(\mathrm{Ar}), 1514$ (Ar), 1602 (Ar), $2875(\mathrm{CH}), 2936(\mathrm{CH}), 3394(\mathrm{NH}) ;{ }^{1} \mathrm{H} \mathrm{NMR}\left(\mathrm{CDCl}_{3}\right): \delta$ $=0.92-0.94\left(\mathrm{t}, 3 \mathrm{H}, \mathrm{CH}_{3}\right), 1.27-1.29\left(\mathrm{~m}, 4 \mathrm{H}, \mathrm{CH}_{2} \mathrm{CH}_{2} \mathrm{CH}_{2} \mathrm{CH}_{3}\right.$ of hexyl group), 1.31-1.35 (m, $2 \mathrm{H}, \mathrm{CH}_{2} \mathrm{CH}_{3}$ of hexyl group), 1.52-1.58 (m, $2 \mathrm{H}, \mathrm{CH}_{2} \cdot \mathrm{CH}_{2} \cdot \mathrm{CH}_{3}$ of hexyl group), 2.0 (bs, $\mathrm{H}$, $\mathrm{NH}$ ), 2.34-2.42 (m, 2H, naphthyl-O- $\mathrm{CH}_{2} \mathrm{CH}_{2} \mathrm{CH}_{2}-\mathrm{S}-\mathrm{C}=\mathrm{S}$ ), 2.63-2.66 (m, 2H, $\mathrm{NHCH}_{2}$ ), 2.81-2.86 (t, 2H, $\left.\mathrm{CH}_{2}-\mathrm{S}-\mathrm{C}=\mathrm{S}\right)$, 4.02-4.05 (t, 2H, $\mathrm{CH}_{2}$-O-naphthyl), 6.97-7.64 (m, 7H, Ar-H of naphthyloxy); Mass: $m / e$ 361; Analysis: $\mathrm{C}_{20} \mathrm{H}_{27} \mathrm{NOS}_{2}$, Calcd. (\%): C, 66.44, H 7.53, N, 3.87, Obsd. (\%): C, 66.75, H, 7.38, $\mathrm{N}, 3.71$.

Hexyl-dithiocarbamic acid-4-(naphthalene-2-yloxy)butyl ester (9): Yield: $0.72 \mathrm{~g}\left(98 \%\right.$ ); m.p.: $129{ }^{\circ} \mathrm{C}$; IR (KBr, $\left.v_{\max }, \mathrm{cm}^{-1}\right)$ : $669(\mathrm{C}-\mathrm{S}), 1116(\mathrm{C}=\mathrm{S}), 1475(\mathrm{Ar}), 1514(\mathrm{Ar}), 1610$ (Ar), $2875(\mathrm{CH}), 2936(\mathrm{CH}), 3410(\mathrm{NH}) ;{ }^{1} \mathrm{H} \mathrm{NMR}\left(\mathrm{CDCl}_{3}\right): \delta$ $=0.92-0.96\left(\mathrm{t}, 3 \mathrm{H}, \mathrm{CH}_{3}\right), 1.25-1.29\left(\mathrm{~m}, 4 \mathrm{H}, \mathrm{CH}_{2} \mathrm{CH}_{2} \mathrm{CH}_{2} \mathrm{CH}_{3}\right.$ of hexyl group), 1.30-1.34 (m, $2 \mathrm{H}, \mathrm{CH}_{2} \mathrm{CH}_{3}$ of hexyl group), 1.53-1.56 (m, $2 \mathrm{H}, \mathrm{CH}_{2} \mathrm{CH}_{2} \mathrm{CH}_{2} \cdot \mathrm{CH}_{2} \cdot \mathrm{CH}_{3}$ of hexyl group), 1.71$1.73\left(\mathrm{~m}, 2 \mathrm{H}\right.$, naphthyl-O- $\left.\mathrm{CH}_{2} \mathrm{CH}_{2}\right), 1.94-1.96(\mathrm{~m}, 2 \mathrm{H}, \mathrm{S}-$ $\mathrm{CH}_{2} \mathrm{CH}_{2}$ ), 2.0 (bs, H, NH), 2.62-2.64 (m, 2H, $\mathrm{NHCH}_{2}$ ), 2.822.86 (t, 2H, CH $2-\mathrm{S}-\mathrm{C}=\mathrm{S}$ ), 4.02-4.06 (t, 2H, $\mathrm{CH}_{2}-\mathrm{O}$-naphthyl), 6.95-7.62 (m, 7H, Ar-H of naphthyloxy); Mass: m/e 375; Analysis: $\mathrm{C}_{21} \mathrm{H}_{29} \mathrm{NOS}_{2}$, Calcd. (\%): C, 67.15, H 7.78, N, 3.73, Obsd. (\%): C, 67.59, H, 7.56, N, 3.51.

Octyl-dithiocarbamic acid-2-(naphthalen-2-yloxy)ethyl ester (10): Yield: 0.85 g, (96.2\%); m.p.: $172{ }^{\circ} \mathrm{C}$; IR (KBr, $\left.v_{\max }, \mathrm{cm}^{-1}\right)$ : $667(\mathrm{C}-\mathrm{S}), 1120(\mathrm{C}=\mathrm{S}), 1475$ (Ar), 1521 (Ar), 1612 (Ar), $2886(\mathrm{CH}), 2941(\mathrm{CH}), 3399(\mathrm{NH}) ;{ }^{1} \mathrm{H} \mathrm{NMR}\left(\mathrm{CDCl}_{3}\right): \delta$ $=0.92-0.94\left(\mathrm{t}, 3 \mathrm{H}, \mathrm{CH}_{3}\right), 1.27-1.29\left(\mathrm{~m}, 8 \mathrm{H}, \mathrm{CH}_{2} \mathrm{CH}_{2} \mathrm{CH}_{2} \mathrm{CH}_{2}\right.$ $\mathrm{CH}_{2} \mathrm{CH}_{3}$ of octyl group), 1.32-1.34 (m, $2 \mathrm{H}, \mathrm{CH}_{2} \mathrm{CH}_{3}$ of octyl group), $1.53-1.56\left(\mathrm{~m}, 2 \mathrm{H}, \mathrm{CH}_{2} \mathrm{CH}_{2} \mathrm{~N}\right.$ of n-octyl group), 2.0 (bs, H, NH), 2.62-2.64 (m, 2H, $\mathrm{NHCH}_{2}$ ), 3.25-3.29 (t, 2H, $\mathrm{CH}_{2}-\mathrm{S}-\mathrm{C}=\mathrm{S}$ ), 4.01-4.04 (t, 2H, $\mathrm{CH}_{2}-\mathrm{O}$-naphthyl), 6.95-7.62 (m, 7H, Ar-H of naphthyloxy); Mass: m/e 375.17; Analysis: $\mathrm{C}_{21} \mathrm{H}_{29} \mathrm{NOS}_{2}$, Calcd. (\%): C, 67.15, H, 7.78, N, 3.73; O, 4.22; S, 17.04.Obsd. (\%): C, 67.15, H, 7.78, N, 3.73, O, 4.26; S, 17.07 .

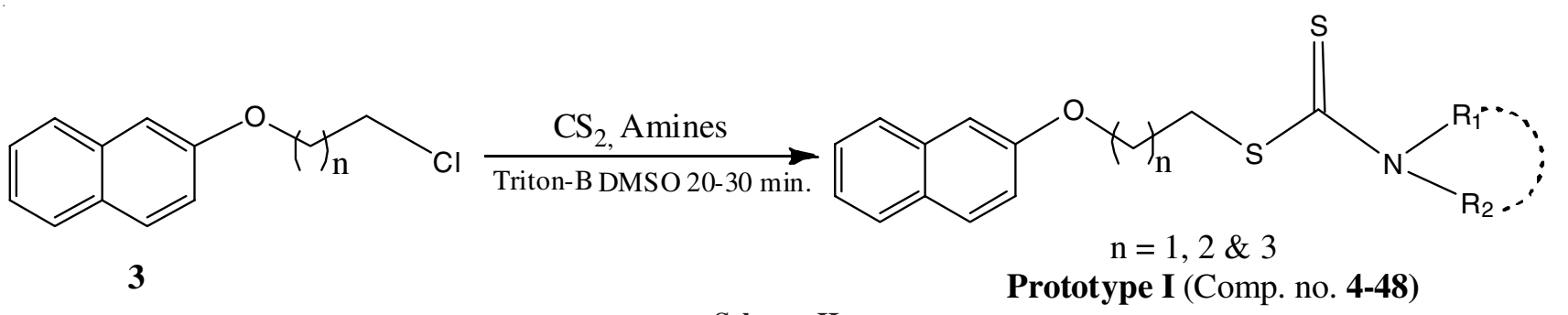

Scheme-II 
Octyl-dithiocarbamic acid-3-(naphthalene-2-yloxy)propyl ester (11): Yield: $0.85 \mathrm{~g},(96.2 \%)$; m.p.: $172{ }^{\circ} \mathrm{C}$; IR $\left(\mathrm{KBr}, v_{\max }, \mathrm{cm}^{-1}\right)$ : $667(\mathrm{C}-\mathrm{S}), 1120(\mathrm{C}=\mathrm{S}), 1472(\mathrm{Ar}), 1521$ (Ar), 1612 (Ar), $2884(\mathrm{CH}), 2941(\mathrm{CH}), 3399(\mathrm{NH}) ;{ }^{1} \mathrm{H}$ NMR $\left(\mathrm{CDCl}_{3}\right): \delta=0.92-0.94\left(\mathrm{t}, 3 \mathrm{H}, \mathrm{CH}_{3}\right), 1.27-1.29(\mathrm{~m}, 8 \mathrm{H}$, $\mathrm{CH}_{2} \mathrm{CH}_{2} \mathrm{CH}_{2} \mathrm{CH}_{2} \mathrm{CH}_{2} \mathrm{CH}_{3}$ of octyl group), $1.30-1.32$ (m, $2 \mathrm{H}$, $\mathrm{CH}_{2} \mathrm{CH}_{3}$ of octyl group), $1.53-1.56\left(\mathrm{~m}, 2 \mathrm{H}, \mathrm{CH}_{2} \mathrm{CH}_{2} \mathrm{~N}\right.$ of $n$ octyl group), 2.0 (bs, H, NH), 2.38-2.42 (m, 2H, naphthyl-O$\left.\mathrm{CH}_{2} \mathrm{CH}_{2} \mathrm{CH}_{2}-\mathrm{S}-\mathrm{C}=\mathrm{S}\right), 2.63-2.66\left(\mathrm{~m}, 2 \mathrm{H}, \mathrm{NHCH}_{2}\right), 2.83-2.87$ (t, 2H, $\mathrm{CH}_{2}$-S-C=S), 4.01-4.04 (t, 2H, $\mathrm{CH}_{2}$-O-naphthyl), 6.987.66 (m, 7H, Ar-H of naphthyloxy); Mass: $\mathrm{m} / \mathrm{e}$ 389; Analysis: $\mathrm{C}_{22} \mathrm{H}_{31} \mathrm{NOS}_{2}$, Calcd. (\%): C, 67.62, H, 8.02, N, 3.59; Obsd. (\%): C, 67.89, H, 7.90, N, 3.44.

Octyl-dithiocarbamic acid-4-(naphthalene-2-yloxy)butyl ester (12): Yield: $0.86 \mathrm{~g}(94 \%)$; m.p.: $156^{\circ} \mathrm{C}$; IR (KBr, $\left.v_{\max }, \mathrm{cm}^{-1}\right): 664(\mathrm{C}-\mathrm{S}), 1108(\mathrm{C}=\mathrm{S}), 1463(\mathrm{Ar}), 1514(\mathrm{Ar}), 1602(\mathrm{Ar})$, $2862(\mathrm{CH}), 2926(\mathrm{CH}), 3392(\mathrm{NH}) ;{ }^{1} \mathrm{H} N \mathrm{MR}\left(\mathrm{CDCl}_{3}\right): \delta=0.93-$ 0.95 (t, 3H, $\left.\mathrm{CH}_{3}\right), 1.27-1.29$ (m, $8 \mathrm{H}, \mathrm{CH}_{2} \mathrm{CH}_{2} \mathrm{CH}_{2} \mathrm{CH}_{2} \mathrm{CH}_{2} \mathrm{CH}_{3}$ of octyl group), 1.30-1.34 (m, $2 \mathrm{H}, \mathrm{CH}_{2} \mathrm{CH}_{3}$ of octyl group), 1.52-1.57 (m, $\left.2 \mathrm{H}, \mathrm{CH}_{2} \cdot \mathrm{CH}_{2} . \mathrm{N}\right), 2.0$ (bs, $\left.\mathrm{H}, \mathrm{NH}\right), 2.63-2.67$ (m, 2H, NHCH $)_{2}$, 3.26-3.32 (t, 2H, $\left.\mathrm{CH}_{2}-\mathrm{S}-\mathrm{C}=\mathrm{S}\right)$, 4.71-4.74 (t, $2 \mathrm{H}, \mathrm{CH}_{2}$-O-naphthyl), 6.96-7.62 (m, 7H, Ar-H of naphthyloxy); Mass: m/e 375; Analysis: $\mathrm{C}_{21} \mathrm{H}_{29} \mathrm{NOS}_{2}$, Calcd. (\%): C, 67.15, H 7.78, N, 3.73, Obsd. (\%): C, 67.54, H, 7.56, N, 3.56.

Decyl-dithiocarbamic acid-2-(naphthalen-2-yloxy)ethyl ester (13): Yield: $0.86 \mathrm{~g}(94 \%)$; m.p.: $156^{\circ} \mathrm{C}$; IR (KBr, $v_{\max }$, $\left.\mathrm{cm}^{-1}\right)$ : 663 (C-S), $1108(\mathrm{C}=\mathrm{S}), 1465$ (Ar), $1511(\mathrm{Ar}), 1605$ (Ar), $2862(\mathrm{CH}), 2926(\mathrm{CH}), 3392(\mathrm{NH}) ;{ }^{1} \mathrm{H}$ NMR $\left(\mathrm{CDCl}_{3}\right): \delta$ $=0.92-0.94\left(\mathrm{t}, 3 \mathrm{H}, \mathrm{CH}_{3}\right), 1.27-1.29\left(\mathrm{~m}, 10 \mathrm{H}, \mathrm{CH}_{2} \mathrm{CH}_{2} \mathrm{CH}_{2} \mathrm{CH}_{2}\right.$ $\mathrm{CH}_{2} \mathrm{CH}_{2} \mathrm{CH}_{2} \mathrm{CH}_{3}$ of decyl group), 1.31-1.35 (m, $2 \mathrm{H}, \mathrm{CH}_{2} \mathrm{CH}_{3}$ of decyl group), $1.53-1.56\left(\mathrm{~m}, 2 \mathrm{H}, \mathrm{CH}_{2} \mathrm{CH}_{2} \mathrm{~N}\right.$ of $n$-decyl group), 2.01 (bs, $\mathrm{H}, \mathrm{NH}), 2.63-2.65\left(\mathrm{~m}, 2 \mathrm{H}, \mathrm{NHCH}_{2}\right), 3.25-$ 3.29 (t, 2H, $\left.\mathrm{CH}_{2}-\mathrm{S}-\mathrm{C}=\mathrm{S}\right), 4.68-4.71$ (t, 2H, $\mathrm{CH}_{2}$-O-naphthyl), 6.97-7.64 (m, 7H, Ar-H of naphthyloxy); Mass: m/e 403.25; Analysis: $\mathrm{C}_{23} \mathrm{H}_{33} \mathrm{NOS}_{2}$, Calcd. (\%): C, 68.40, H 8.20, N, 3.90, O, 3.92, S, 15.86; Obsd. (\%): C, 68.44, H, 8.24, N, 3.96. O, 3.96, S, 15.89.

Decyl-dithiocarbamic acid-3-(naphthalene-2-yloxy)propyl ester (14): Yield: $0.86 \mathrm{~g}$ (94\%); m.p.: $156{ }^{\circ} \mathrm{C}$; IR $\left(\mathrm{KBr}, v_{\max }, \mathrm{cm}^{-1}\right)$ : $664(\mathrm{C}-\mathrm{S}), 1109(\mathrm{C}=\mathrm{S}), 1462$ (Ar), 1513 (Ar), 1602 (Ar), $2863(\mathrm{CH}), 2925(\mathrm{CH}), 3392(\mathrm{NH}) ;{ }^{1} \mathrm{H}$ NMR $\left(\mathrm{CDCl}_{3}\right): \delta=0.92-0.95\left(\mathrm{t}, 3 \mathrm{H}, \mathrm{CH}_{3}\right), 1.27-1.29(\mathrm{~m}, 10 \mathrm{H}$, $\mathrm{CH}_{2} \mathrm{CH}_{2} \mathrm{CH}_{2} \mathrm{CH}_{2} \mathrm{CH}_{2} \mathrm{CH}_{2} \mathrm{CH}_{2} \mathrm{CH}_{3}$ of decyl group), 1.31-1.35 (m, $2 \mathrm{H}, \mathrm{CH}_{2} \mathrm{CH}_{3}$ of decyl group), 1.53-1.56 (m, $2 \mathrm{H}, \mathrm{CH}_{2} \mathrm{CH}_{2} \mathrm{~N}$ of $n$-decyl group), 2.0 (bs, $\mathrm{H}, \mathrm{NH}), 2.62-2.66\left(\mathrm{~m}, 2 \mathrm{H}, \mathrm{NHCH}_{2}\right)$, 2.84-2.87 (t, $\left.2 \mathrm{H}, \mathrm{CH}_{2}-\mathrm{S}-\mathrm{C}=\mathrm{S}\right), 2.34-2.38\left(\mathrm{~m}, 2 \mathrm{H}, \mathrm{CH}_{2} \mathrm{CH}_{2}\right.$ ), 4.68-4.71 (t, 2H, $\mathrm{CH}_{2}$-O-naphthyl), 6.96-7.62 (m, 7H, Ar-H of naphthyloxy); Mass: m/e 417.67; Analysis: $\mathrm{C}_{24} \mathrm{H}_{35} \mathrm{NOS}_{2}$, Calcd. (\%): C, 69, H 8.40, N, 3.35, O, 3.80, S, 15.31. O, 3.83, S, 15.35. Obsd. (\%): C, 69.02, H, 8.45, N, 3.35, O, 3.83, S, 15.35.

Decyl-dithiocarbamic acid-4-(naphthalene-2-yloxy)butyl ester (15): Yield: $0.86 \mathrm{~g}(94 \%)$; m.p.: $156^{\circ} \mathrm{C}$; IR (KBr, $\left.v_{\max }, \mathrm{cm}^{-1}\right): 663(\mathrm{C}-\mathrm{S}), 1107(\mathrm{C}=\mathrm{S}), 1462(\mathrm{Ar}), 1510(\mathrm{Ar}), 1606$ (Ar), $2865(\mathrm{CH}), 2926(\mathrm{CH}), 3392(\mathrm{NH}) ;{ }^{1} \mathrm{H} \mathrm{NMR}\left(\mathrm{CDCl}_{3}\right)$ : $\delta$ $=0.92-0.94\left(\mathrm{t}, 3 \mathrm{H}, \mathrm{CH}_{3}\right), 1.27-1.29\left(\mathrm{~m}, 10 \mathrm{H}, \mathrm{CH}_{2} \mathrm{CH}_{2} \mathrm{CH}_{2} \mathrm{CH}_{2}\right.$ $\mathrm{CH}_{2} \mathrm{CH}_{2} \mathrm{CH}_{2} \mathrm{CH}_{3}$ of decyl group), 1.30-1.34 (m, 2H, $\mathrm{CH}_{2} \mathrm{CH}_{3}$ of decyl group), 1.52-1.58 (m, $2 \mathrm{H}, \mathrm{CH}_{2} \mathrm{CH}_{2} \mathrm{~N}$ of $n$-decyl group), 2.0 (bs, H, NH), 2.62-2.66 (m, $\left.2 \mathrm{H}, \mathrm{NHCH}_{2}\right), 2.84-$
2.87 (t, 2H, $\left.\mathrm{CH}_{2}-\mathrm{S}-\mathrm{C}=\mathrm{S}\right), 1.93-1.95\left(\mathrm{~m}, 2 \mathrm{H}, \mathrm{CH}_{2} \mathrm{CH}_{2} \mathrm{CH}_{2}\right.$ ), 1.67-1.71 (m, 2H, $\mathrm{CH}_{2} \mathrm{CH}_{2} \mathrm{CH}_{2}$ ), 4.68-4.71 (t, $2 \mathrm{H}, \mathrm{CH}_{2}$-Onaphthyl), 6.97-7.64 (m, 7H, Ar-H of naphthyloxy); Mass: m/e 431.70; Analysis: $\mathrm{C}_{25} \mathrm{H}_{37} \mathrm{NOS}_{2}$, Calcd. (\%): C, 69.52, H 8.63, N, 3.21, O, 3.70, S, 14.82. Obsd. (\%): C, 69.56, H, 8.64, N, 3.24, O, 3.71, S, 14.86.

Pyrrolidine-dithiocarbamic acid-2-(naphthalen-2yloxy)ethyl ester (16): Yield: $0.62 \mathrm{~g}(80.8 \%)$; m.p.: $79^{\circ} \mathrm{C}$; IR (KBr, $\left.v_{\max }, \mathrm{cm}^{-1}\right): 657(\mathrm{C}-\mathrm{S}), 1106(\mathrm{C}=\mathrm{S}), 1454(\mathrm{Ar}), 1502$ (Ar), 1600 (Ar), $2863(\mathrm{CH}), 2925(\mathrm{CH}) ;{ }^{1} \mathrm{H} \mathrm{NMR}\left(\mathrm{CDCl}_{3}\right)$ : $\delta$ $=1.58-1.60\left(\mathrm{~m}, 4 \mathrm{H}, \mathrm{CH}_{2}\right.$ of pyrrolidine ring $), 2.8\left(\mathrm{t}, 4 \mathrm{H}, \mathrm{CH}_{2} \mathrm{~N}\right.$ of pyrrolidine ring $\left(2 \mathrm{H}, \mathrm{CH}_{2}-\mathrm{S}-\mathrm{C}=\mathrm{S}\right), 4.71-4.73\left(\mathrm{t}, 2 \mathrm{H}, \mathrm{CH}_{2^{-}}\right.$ O-naphthyl), 6.96-7.62 (m, 7H, Ar-H of naphthyloxy); Mass: m/e 317; Analysis: $\mathrm{C}_{17} \mathrm{H}_{19} \mathrm{NOS}_{2}$, Calcd. (\%): C, 64.32, H, 6.03, N, 4.41, Obsd. (\%): C, 63.85, H, 6.29, N, 4.64.

Pyrrolidine-dithiocarbamic acid-3-(naphthalen-2yloxy)propyl ester (17): Yield: $0.63 \mathrm{~g}(83.2 \%)$; m.p.: $86^{\circ} \mathrm{C}$; IR (KBr, $\left.v_{\max }, \mathrm{cm}^{-1}\right)$ : $672(\mathrm{C}-\mathrm{S}), 1124(\mathrm{C}=\mathrm{S}), 1475(\mathrm{Ar}), 1524$ (Ar), $1605(\mathrm{Ar}), 2885(\mathrm{CH}), 2926(\mathrm{CH}) ;{ }^{1} \mathrm{H}$ NMR $\left(\mathrm{CDCl}_{3}\right): \delta$ $=1.57-1.61\left(\mathrm{~m}, 4 \mathrm{H}, \mathrm{CH}_{2}\right.$ of pyrrolidine ring $), 2.35-2.38(\mathrm{~m}$, $2 \mathrm{H}$, naphthyl-O- $\mathrm{CH}_{2} \mathrm{CH}_{2} \mathrm{CH}_{2}-\mathrm{S}-\mathrm{C}=\mathrm{S}$ ), 2.8 (t, $4 \mathrm{H}, \mathrm{CH}_{2} \mathrm{~N}$ of pyrrolidine ring), 2.82-2.86 ( $\left.\mathrm{t}, 2 \mathrm{H}, \mathrm{CH}_{2}-\mathrm{S}-\mathrm{C}=\mathrm{S}\right), 4.02-4.04$ (t, 2H, $\mathrm{CH}_{2}$-O-naphthyl), 6.96-7.62 (m, 7H, Ar-H of naphthyloxy); Mass: m/e 331; Analysis: $\mathrm{C}_{18} \mathrm{H}_{21} \mathrm{NOS}_{2}$, Calcd. (\%): C, 65.22, H, 6.39, N, 4.23, Obsd. (\%): C, 65.63, H, 6.12, N, 4.01.

Pyrrolidine-dithiocarbamic acid-4-(naphthalen-2yloxy)butyl ester (18): Yield: $0.64 \mathrm{~g}(86.5 \%)$; m.p.: $95^{\circ} \mathrm{C}$; IR $\left(\mathrm{KBr}, v_{\max }, \mathrm{cm}^{-1}\right)$ : $679(\mathrm{C}-\mathrm{S}), 1125(\mathrm{C}=\mathrm{S}), 1484(\mathrm{Ar}), 1525$ (Ar), $1610(\mathrm{Ar}), 2885(\mathrm{CH}), 2947(\mathrm{CH}) ;{ }^{1} \mathrm{H}$ NMR $\left(\mathrm{CDCl}_{3}\right)$ : $\delta$ $=1.56-1.60\left(\mathrm{~m}, 4 \mathrm{H}, \mathrm{CH}_{2}\right.$ of pyrrolidine ring $), 1.71-1.72(\mathrm{~m}$, $2 \mathrm{H}$, naphthyl-O- $\left.\mathrm{CH}_{2} \mathrm{CH}_{2}\right), 1.95-1.98\left(\mathrm{~m}, 2 \mathrm{H}, \mathrm{S}_{-} \mathrm{CH}_{2} \mathrm{CH}_{2}\right), 2.8$ (t, $4 \mathrm{H}, \mathrm{CH}_{2} \mathrm{~N}$ of pyrrolidine ring), 2.84-2.88 (t, $2 \mathrm{H}, \mathrm{CH}_{2}-\mathrm{S}-$ $\mathrm{C}=\mathrm{S}$ ), 4.02-4.05 (t, 2H, $\mathrm{CH}_{2}-\mathrm{O}$-naphthyl), 6.97-7.64 (m, 7H, Ar-H of naphthyloxy); Mass: m/e 345; Analysis: $\mathrm{C}_{19} \mathrm{H}_{23} \mathrm{NOS}_{2}$, Calcd. (\%): C, 66.09, H, 6.57, N, 4.15, Obsd. (\%): C, 66.57, H, 6.32, N, 3.86.

Piperidine-1-dithiocarbamic acid-2-(naphthalen-2yloxy)ethyl ester (19): Yield: $0.66 \mathrm{~g}(82.5 \%)$; m.p.: $89^{\circ} \mathrm{C}$; IR (KBr, $\left.v_{\max }, \mathrm{cm}^{-1}\right)$ : $660(\mathrm{C}-\mathrm{S}), 1110(\mathrm{C}=\mathrm{S}), 1461(\mathrm{Ar}), 1509$ (Ar), 1605 (Ar), $2859(\mathrm{CH}), 2926(\mathrm{CH}) ;{ }^{1} \mathrm{H} \mathrm{NMR}\left(\mathrm{CDCl}_{3}\right): \delta$ $=1.48-1.50\left(\mathrm{~m}, 6 \mathrm{H}, \mathrm{CH}_{2}\right.$ of piperidine ring $), 2.7\left(\mathrm{t}, 4 \mathrm{H}, \mathrm{CH}_{2} \mathrm{~N}\right.$ of piperidine ring), 3.28-3.30 (t, $2 \mathrm{H}, \mathrm{CH}_{2}-\mathrm{S}-\mathrm{C}=\mathrm{S}$ ), 4.71-4.73 (t, 2H, $\mathrm{CH}_{2}-\mathrm{O}$-naphthyl), 6.95-7.62 (m, 7H, Ar-H of naphthyloxy); Mass: m/e 331; Analysis: $\mathrm{C}_{18} \mathrm{H}_{21} \mathrm{NOS}_{2}$, Calcd. (\%): C, 65.22, H 6.39, N, 4.23, Obsd. (\%): C, 65.73, H, 6.13, N, 3.98 .

Piperidine-1-dithiocarbamic acid-3-(naphthalen-2yloxy)propyl ester (20): Yield: $0.66 \mathrm{~g}(82.5 \%)$; m.p.: $89^{\circ} \mathrm{C}$; IR (KBr, $\left.v_{\max }, \mathrm{cm}^{-1}\right)$ : $662(\mathrm{C}-\mathrm{S}), 1112(\mathrm{C}=\mathrm{S}), 1461(\mathrm{Ar}), 1509$ (Ar), 1605 (Ar), $2859(\mathrm{CH}), 2927(\mathrm{CH}) ;{ }^{1} \mathrm{H}$ NMR $\left(\mathrm{CDCl}_{3}\right): \delta$ $=1.48-1.50\left(\mathrm{~m}, 6 \mathrm{H}, \mathrm{CH}_{2}\right.$ of piperidine ring), $2.7\left(\mathrm{t}, 4 \mathrm{H}, \mathrm{CH}_{2} \mathrm{~N}\right.$ of piperidine ring), 2.82-2.86 (t, $2 \mathrm{H}, \mathrm{CH}_{2}-\mathrm{S}-\mathrm{C}=\mathrm{S}$ ), 2.35-2.38 (m, 2H, $\mathrm{CH}_{2} \mathrm{CH}_{2}$ ), 4.01-4.04 (t, 2H, $\mathrm{CH}_{2}$-O-naphthyl), 6.957.62 (m, 7H, Ar-H of naphthyloxy); Mass: m/e 392.54; Analysis: $\mathrm{C}_{19} \mathrm{H}_{24} \mathrm{~N}_{2} \mathrm{O}_{2} \mathrm{~S}_{3}$, Calcd. (\%): C, 58.22, H 6.20, N, 7.23, Obsd. (\%): C, 58.14.73, H, 6.16, N, 7.14, O, 12.23, S, 16.13.

Piperidine-1-dithiocarbamic acid-4-(naphthalen-2yloxy)butyl ester (21): Yield: $0.66 \mathrm{~g}(82.5 \%)$; m.p.: $89^{\circ} \mathrm{C}$; 
IR (KBr, $\left.v_{\max }, \mathrm{cm}^{-1}\right)$ : $660(\mathrm{C}-\mathrm{S}), 1110(\mathrm{C}=\mathrm{S}), 1461(\mathrm{Ar}), 1509$ (Ar), $1605(\mathrm{Ar}), 2859(\mathrm{CH}), 2925(\mathrm{CH}) ;{ }^{1} \mathrm{H}$ NMR $\left(\mathrm{CDCl}_{3}\right)$ : $\delta$ $=1.48-1.50\left(\mathrm{~m}, 6 \mathrm{H}, \mathrm{CH}_{2}\right.$ of piperidine ring $), 2.7\left(\mathrm{t}, 4 \mathrm{H}, \mathrm{CH}_{2} \mathrm{~N}\right.$ of piperidine ring), 2.83-2.86 (t, $\left.2 \mathrm{H}, \mathrm{CH}_{2}-\mathrm{S}-\mathrm{C}=\mathrm{S}\right), 1.92-1.96$ (m, $2 \mathrm{H}, \mathrm{CH}_{2} \mathrm{CH}_{2}$ ), 1.68-1.71 (m, 2H, $\mathrm{CH}_{2} \mathrm{CH}_{2}$ ), 4.00-4.02 (t, $2 \mathrm{H}, \mathrm{CH}_{2}$-O-naphthyl), 6.96-7.65 (m, 7H, Ar-H of naphthyloxy); Mass: $m / e$ 406.56; Analysis: $\mathrm{C}_{20} \mathrm{H}_{26} \mathrm{~N}_{2} \mathrm{O}_{3} \mathrm{~S}_{2}$, Calcd. (\%): C, 59.12, H 6.49, N, 6.82, O, 11.82, S, 15.75. Obsd. (\%): C, 59.08, H, 6.45, N, 6.89, O, 11.89, S, 15.79.

4-Methyl-piperazine-dithiocarbamic acid-2-(naphthalen2-yloxy)ethyl ester (22): Yield: $0.66 \mathrm{~g}\left(82.5 \%\right.$ ); m.p.: $89^{\circ} \mathrm{C}$; IR (KBr, $\left.v_{\max }, \mathrm{cm}^{-1}\right)$ : $662(\mathrm{C}-\mathrm{S}), 1112(\mathrm{C}=\mathrm{S}), 1461(\mathrm{Ar}), 1509$ (Ar), 1605 (Ar), $2859(\mathrm{CH}), 2926(\mathrm{CH}) ;{ }^{1} \mathrm{H}$ NMR $\left(\mathrm{CDCl}_{3}\right): \delta$ $=2.24-2.27\left(\mathrm{~s}, 3 \mathrm{H}, \mathrm{CH}_{3}\right.$ of methyl piperazine ring $), 2.44-2.48$ (t, $\mathrm{CH}_{2} \mathrm{~N}$ of piperazine ring), 2.62-2.65 ( $\mathrm{t}, \mathrm{CH}_{2} \mathrm{~N}$ of piperazine ring), 2.0 (bs, $\mathrm{H}, \mathrm{NH}$ ), 3.25-3.29 (t, $2 \mathrm{H}, \mathrm{CH}_{2}-\mathrm{S}-\mathrm{C}=\mathrm{S}$ ), 4.684.71 (t, 2H, $\mathrm{CH}_{2}-\mathrm{O}$-naphthyl), 6.96-7.65 (m, 7H, Ar-H of naphthyloxy); Mass: m/e 361; Analysis: $\mathrm{C}_{18} \mathrm{H}_{23} \mathrm{~N}_{3} \mathrm{OS}_{2}$, Calcd. (\%): C, 59.84, H 6.39, N, 11.60, O, 4.40, S, $17.72 \%$ Obsd. (\%): C, 59.80, H, 6.41, N, $11.62 \%$ O, 4.43, S, 17.74.

4-Methyl-piperazine-dithiocarbamic acid-3-(naphthalen2-yloxy)propyl ester (23): Yield: $0.66 \mathrm{~g}(82.5 \%)$; m.p.: 89 ${ }^{\circ} \mathrm{C}$; IR (KBr, $\left.v_{\max }, \mathrm{cm}^{-1}\right)$ : $660(\mathrm{C}-\mathrm{S}), 1110(\mathrm{C}=\mathrm{S}), 1461(\mathrm{Ar})$, 1509 (Ar), 1605 (Ar), 2859 (CH), $2926(\mathrm{CH}) ;{ }^{1} \mathrm{H}$ NMR $\left(\mathrm{CDCl}_{3}\right): \delta=2.24-2.27$ (s, $3 \mathrm{H}, \mathrm{CH}_{3}$ of methyl piperazine ring), 2.44-2.48 ( $\mathrm{t}, \mathrm{CH}_{2} \mathrm{~N}$ of piperazine ring), 2.62-2.65 ( $\mathrm{t}, \mathrm{CH}_{2} \mathrm{~N}$ of piperazine ring), 2.0 (bs, $\mathrm{H}, \mathrm{NH}$ ), 2.84-2.87 (t, $2 \mathrm{H}, \mathrm{CH}_{2}-\mathrm{S}-$ $\mathrm{C}=\mathrm{S}), 2.34-2.38\left(\mathrm{~m}, 2 \mathrm{H}, \mathrm{CH}_{2} \mathrm{CH}_{2}\right), 4.68-4.71$ (t, $2 \mathrm{H}, \mathrm{CH}_{2}-\mathrm{O}-$ naphthyl), 6.96-7.63 (m, 7H, Ar-H of naphthyloxy); Mass: m/e 375.55; Analysis: $\mathrm{C}_{19} \mathrm{H}_{25} \mathrm{~N}_{3} \mathrm{OS}_{2}$, Calcd. (\%): C, 60.72, $\mathrm{H}$ 6.69, N, 11.23, O, 4.22, S, $17.05 \%$ Obsd. (\%): C, 60.76, H, 6.71, N, 11.19, O, 4.26, S, 17.08.

4-Methyl-piperazine-dithiocarbamic acid-4-(naphthalen2-yloxy)butyl ester (24): Yield: $0.66 \mathrm{~g}(82.5 \%)$; m.p.: $89^{\circ} \mathrm{C}$; IR (KBr, $\left.v_{\max }, \mathrm{cm}^{-1}\right): 662(\mathrm{C}-\mathrm{S}), 1112(\mathrm{C}=\mathrm{S}), 1461(\mathrm{Ar}), 1509$ (Ar), 1605 (Ar), $2859(\mathrm{CH}), 2925(\mathrm{CH}) ;{ }^{1} \mathrm{H} \mathrm{NMR}\left(\mathrm{CDCl}_{3}\right)$ : $\delta$ $=2.24-2.27\left(\mathrm{~s}, 3 \mathrm{H}, \mathrm{CH}_{3}\right.$ of methyl piperazine ring), 2.44-2.48 ( $\mathrm{t}, \mathrm{CH}_{2} \mathrm{~N}$ of piperazine ring), 2.62-2.65 ( $\mathrm{t}, \mathrm{CH}_{2} \mathrm{~N}$ of piperazine ring), 2.0 (bs, H, NH), 2.84-2.86 (t, 2H, $\mathrm{CH}_{2}-\mathrm{S}-\mathrm{C}=\mathrm{S}$ ), 1.941.96 (m, $2 \mathrm{H}, \mathrm{CH}_{2} \mathrm{CH}_{2} \mathrm{CH}_{2}$ ), 1.68-1.71 (m, $2 \mathrm{H}, \mathrm{CH}_{2} \mathrm{CH}_{2} \mathrm{CH}_{2}$ ), 4.68-4.71 (t, 2H, $\mathrm{CH}_{2}$-O-naphthyl), 6.96-7.62 (m, 7H, Ar-H of naphthyloxy); Mass: m/e 389.58; Analysis: $\mathrm{C}_{20} \mathrm{H}_{27} \mathrm{~N}_{3} \mathrm{OS}_{2}$, Calcd. (\%): C, 61.64, H 6.93, N, 10.73, O, 4.08, S, $16.42 \%$ Obsd. (\%): C, 61.66, H, 6.99, N, 10.79, O, 4.11, S, 16.46.

Morpholine 4-dithiocarbamic acid-2-(naphthalen-2yloxy)ethyl ester (25): Yield: $0.66 \mathrm{~g}(82.5 \%)$; m.p.: $89^{\circ} \mathrm{C}$; IR (KBr, $\left.v_{\max }, \mathrm{cm}^{-1}\right): 660(\mathrm{C}-\mathrm{S}), 1110(\mathrm{C}=\mathrm{S}), 1461(\mathrm{Ar}), 1509$ (Ar), $1605(\mathrm{Ar}), 2859(\mathrm{CH}), 2926(\mathrm{CH}) ;{ }^{1} \mathrm{H} \mathrm{NMR}\left(\mathrm{CDCl}_{3}\right): \delta$ $=3.62-3.67\left(\mathrm{t}, 2 \mathrm{H}, \mathrm{CH}_{2}\right.$ of morphaline ring), 2.34-2.37 (t, $\mathrm{CH}_{2} \mathrm{~N}$ of morphaline ring), 2.35-2.40 (s, $\left.\mathrm{CH}_{2} \mathrm{SCS}\right), 2.84-2.86$ (t, $2 \mathrm{H}$, $\left.\mathrm{CH}_{2}-\mathrm{S}-\mathrm{C}=\mathrm{S}\right), 2.34-2.38$ (m, 2H, $\left.\mathrm{CH}_{2} \mathrm{CH}_{2}\right), 4.68-4.71$ (t, $2 \mathrm{H}$, $\mathrm{CH}_{2}$-O-naphthyl), 6.95-7.62 (m, 7H, Ar-H of naphthyloxy); Mass: m/e 382.50; Analysis: $\mathrm{C}_{17} \mathrm{H}_{22} \mathrm{~N}_{2} \mathrm{O}_{4} \mathrm{~S}_{2}$, Calcd. (\%): $\mathrm{C}$, 53.35, H 5.79, N, 7.30, O, 16.70, S, 16.72 Obsd. (\%): C, 53.38, H, 5.80, N, 7.32, O, 16.73, S, 16.77.

Morpholine 4-dithiocarbamic acid-3-(naphthalen-2yloxy)propyl ester (26): Yield: $0.66 \mathrm{~g}(82.5 \%)$; m.p.: $89^{\circ} \mathrm{C}$; IR (KBr, $\left.v_{\max }, \mathrm{cm}^{-1}\right)$ : $662(\mathrm{C}-\mathrm{S}), 1112(\mathrm{C}=\mathrm{S}), 1461(\mathrm{Ar}), 1509$
(Ar), $1605(\mathrm{Ar}), 2859(\mathrm{CH}), 2926(\mathrm{CH}) ;{ }^{1} \mathrm{H}$ NMR $\left(\mathrm{CDCl}_{3}\right)$ : $\delta$ $=3.62-3.67\left(\mathrm{t}, 2 \mathrm{H}, \mathrm{CH}_{2}\right.$ of morphaline ring), 2.34-2.37 (t, $\mathrm{CH}_{2} \mathrm{~N}$ of morphaline ring), 2.35-2.40 (s, $\left.\mathrm{CH}_{2} \mathrm{~S}-\mathrm{C}=\mathrm{S}\right), 1.95-1.99$ (m, $4 \mathrm{H}, \mathrm{CH}_{2} \mathrm{CH}_{2}$ ), 4.68-4.71 (t, 2H, $\mathrm{CH}_{2}$-O-naphthyl), 6.96-7.65 (m, 7H, Ar-H of naphthyloxy); Mass: m/e 396.52; Analysis: $\mathrm{C}_{18} \mathrm{H}_{24} \mathrm{~N}_{2} \mathrm{O}_{4} \mathrm{~S}_{2}$, Calcd. (\%): C, 45.50, H 6.09, N, 7.10, O, 16.10, S, 16.15. Obsd. (\%): C, 45.52, H, 6.10, N, 7.06\% O, 16.14, S, 16.17 .

Morpholine 4-dithiocarbamic acid-4-(naphthalen-2yloxy)butyl ester (27): Yield: $0.66 \mathrm{~g}(82.5 \%)$; m.p.: $89^{\circ} \mathrm{C}$; IR (KBr, $\left.v_{\max }, \mathrm{cm}^{-1}\right)$ : $660(\mathrm{C}-\mathrm{S}), 1110(\mathrm{C}=\mathrm{S}), 1461(\mathrm{Ar}), 1509$ (Ar), $1605(\mathrm{Ar}), 2859(\mathrm{CH}), 2926(\mathrm{CH}) ;{ }^{1} \mathrm{H}$ NMR $\left(\mathrm{CDCl}_{3}\right): \delta$ $=3.62-3.67\left(\mathrm{t}, 2 \mathrm{H}, \mathrm{CH}_{2}\right.$ of morphaline ring), 2.34-2.37 $\left(\mathrm{t}, \mathrm{CH}_{2} \mathrm{~N}\right.$ of morphaline ring), 2.35-2.40 (s, $\mathrm{CH}_{2} \mathrm{SCS}$ ), 3.25-3.28 (t, $2 \mathrm{H}$, $\mathrm{CH}_{2}$-S-C=S), 1.94-1.96 (m, 2H, $\mathrm{CH}_{2} \mathrm{CH}_{2} \mathrm{CH}_{2}$ ), 1.68-1.71 (m, $2 \mathrm{H}, \mathrm{CH}_{2} \mathrm{CH}_{2} \mathrm{CH}_{2}$ ), 4.68-4.71 (t, 2H, $\mathrm{CH}_{2}$-O-naphthyl), 6.967.63 (m, 7H, Ar-H of naphthyloxy); Mass: m/e 410.55; Analysis: $\mathrm{C}_{19} \mathrm{H}_{26} \mathrm{~N}_{2} \mathrm{O}_{4} \mathrm{~S}_{2}$, Calcd. (\%): C, 55.55, H 6.39, N, 6.30, O, 15.55, S, 15.60. Obsd. (\%): C, 55.58, H, 6.35, N, 6.85, O, 15.59, S, 15.62 .

p-Tolyl-dithiocarbamic acid-2-(naphthalen-2-yloxy)ethyl ester (28): Yield: $0.76 \mathrm{~g}\left(88.4 \%\right.$ ); m.p.: $137^{\circ} \mathrm{C}$; $\mathrm{IR}(\mathrm{KBr}$, $\left.v_{\max }, \mathrm{cm}^{-1}\right): 660(\mathrm{C}-\mathrm{S}), 1111(\mathrm{C}=\mathrm{S}), 1454(\mathrm{Ar}), 1502(\mathrm{Ar}), 1602$ $(\mathrm{Ar}), 2851(\mathrm{CH}), 2928(\mathrm{CH}), 3388(\mathrm{NH}) ;{ }^{1} \mathrm{H} \mathrm{NMR}\left(\mathrm{CDCl}_{3}\right): \delta$ $=2.34\left(\mathrm{~s}, 3 \mathrm{H}, \mathrm{CH}_{3}\right), 3.28-3.30\left(\mathrm{t}, 2 \mathrm{H}, \mathrm{CH}_{2}-\mathrm{S}-\mathrm{C}=\mathrm{S}\right), 4.0(\mathrm{bs}, \mathrm{H}$, $\mathrm{NH}), 4.70-4.72$ (t, 2H, $\mathrm{CH}_{2}$-O-naphthyl), 6.35-7.62 (m, 11H, Ar-H of naphthyloxy and phenyl ring); Mass: m/e 353; Analysis: $\mathrm{C}_{20} \mathrm{H}_{19} \mathrm{NOS}_{2}$, Calcd. (\%): C, 67.89, H, 5.45, N, 3.99, Obsd. (\%): C, 67.63, H, 5.58, N, 4.12.

p-Tolyl-dithiocarbamic acid-3-(naphthalen-2-yloxy)propyl ester (29): Yield: $0.76 \mathrm{~g}(88.4 \%)$; m.p.: $137^{\circ} \mathrm{C}$; IR $\left(\mathrm{KBr}, v_{\max }, \mathrm{cm}^{-1}\right)$ : $662(\mathrm{C}-\mathrm{S}), 1111(\mathrm{C}=\mathrm{S}), 1454(\mathrm{Ar}), 1502$ (Ar), 1601 (Ar), $2851(\mathrm{CH}), 2928(\mathrm{CH}), 3388(\mathrm{NH}) ;{ }^{1} \mathrm{H}$ NMR $\left(\mathrm{CDCl}_{3}\right): \delta=2.34\left(\mathrm{~s}, 3 \mathrm{H}, \mathrm{CH}_{3}\right), 3.28-3.30$ (t, $2 \mathrm{H}, \mathrm{CH}_{2}-\mathrm{S}-\mathrm{C}=\mathrm{S}$ ), 4.0 (bs, H, NH), 2.35-2.40 (s, $\left.\mathrm{CH}_{2} \mathrm{~S}-\mathrm{C}=\mathrm{S}\right), 1.95-1.99$ (m, $4 \mathrm{H}$, $\mathrm{CH}_{2} \mathrm{CH}_{2}$ ), 4.68-4.71 (t, $2 \mathrm{H}, \mathrm{CH}_{2}$-O-naphthyl), 6.34-7.64 (m, $11 \mathrm{H}, \mathrm{Ar}-\mathrm{H}$ of naphthyloxy and phenyl ring); Mass: $\mathrm{m} / \mathrm{e} 367.53$; Analysis: $\mathrm{C}_{21} \mathrm{H}_{21} \mathrm{NOS}_{2}$, Calcd. (\%): C, 68.60, H, 5.72, N, 3.80, O, 4.31, S, $17.45 \%$ Obsd. (\%): C, 68.63, H, 5.76, N, $3.81 \%$ $\mathrm{O}, 4.35, \mathrm{~S}, 17.45$.

p-Tolyl-dithiocarbamic acid-4-(naphthalen-2-yloxy)butyl ester (30): Yield: $0.76 \mathrm{~g}(88.4 \%)$; m.p.: $137^{\circ} \mathrm{C}$; IR (KBr, $\left.v_{\max }, \mathrm{cm}^{-1}\right): 662(\mathrm{C}-\mathrm{S}), 1111(\mathrm{C}=\mathrm{S}), 1454(\mathrm{Ar}), 1502(\mathrm{Ar}), 1601$ (Ar), $2851(\mathrm{CH}), 2928(\mathrm{CH}), 3388(\mathrm{NH}) ;{ }^{1} \mathrm{H}$ NMR $\left(\mathrm{CDCl}_{3}\right): \delta$ $=2.34\left(\mathrm{~s}, 3 \mathrm{H}, \mathrm{CH}_{3}\right), 3.28-3.30\left(\mathrm{t}, 2 \mathrm{H}, \mathrm{CH}_{2}-\mathrm{S}-\mathrm{C}=\mathrm{S}\right), 4.0(\mathrm{bs}, \mathrm{H}$, $\mathrm{NH}$ ), 2.35-2.40 (s, $\mathrm{CH}_{2} \mathrm{SCS}$ ), 3.25-3.27 (t, $\left.2 \mathrm{H}, \mathrm{CH}_{2}-\mathrm{S}-\mathrm{C}=\mathrm{S}\right)$, 1.94-1.96 (m, 2H, $\left.\mathrm{CH}_{2} \mathrm{CH}_{2} \mathrm{CH}_{2}\right), 1.68-1.71$ ( $\mathrm{m}, 2 \mathrm{H}, \mathrm{CH}_{2} \mathrm{CH}_{2} \mathrm{CH}_{2}$ ), 4.71-4.73 (t, 2H, $\mathrm{CH}_{2}$-O-naphthyl), 6.35-7.63 (m, 11H, Ar-H of naphthyloxy and phenyl ring); Mass: $m / e$ 381.55; Analysis: $\mathrm{C}_{22} \mathrm{H}_{23} \mathrm{NOS}_{2}$, Calcd. (\%): C, 69.20, H, 6.06, N, 3.62, O, 4.15, S, 16.79. Obsd. (\%): C, 69.25, H, 6.08, N, 3.67, O, 4.19, S, 16.81 .

(4-Methoxy-4-phenyl)dithiocarbamic acid-2-(naphthalen2-yloxy)ethyl ester (31): Yield: $0.8 \mathrm{~g}(89.2 \%)$; m.p.: $117^{\circ} \mathrm{C}$; IR (KBr, $\left.v_{\max }, \mathrm{cm}^{-1}\right): 659(\mathrm{C}-\mathrm{S}), 1106(\mathrm{C}=\mathrm{S}), 1455(\mathrm{Ar}), 1502$ (Ar), 1600 (Ar), $2854(\mathrm{CH}), 2926(\mathrm{CH}), 3389(\mathrm{NH}) ;{ }^{1} \mathrm{H}$ NMR $\left(\mathrm{CDCl}_{3}\right): \delta=3.28-3.30\left(\mathrm{t}, 2 \mathrm{H}, \mathrm{CH}_{2}-\mathrm{S}-\mathrm{C}=\mathrm{S}\right), 3.72(\mathrm{~s}, 3 \mathrm{H}$, $\mathrm{OCH}_{3}$ ), 4.0 (bs, H, NH), 4.70-4.72 (t, 2H, $\mathrm{CH}_{2}$-O-naphthyl), 
6.35-7.64 (m, 11H, Ar-H of naphthyloxy and phenyl ring); Mass: m/e 369; Analysis: $\mathrm{C}_{20} \mathrm{H}_{19} \mathrm{NO}_{2} \mathrm{~S}_{2}$, Calcd. (\%): C, 65.01, H, 5.18, N, 3.79, Obsd. (\%): C, 65.47, H, 5.03, N, 3.48 .

(4-Methoxy-4-phenyl)dithiocarbamic acid-3-(naphthalen2-yloxy)propyl ester (32): Yield: 0.82 g (93.8 \%); m.p.: 139 ${ }^{\circ} \mathrm{C}$; IR (KBr, $\left.v_{\max }, \mathrm{cm}^{-1}\right)$ : 668 (C-S), 1117 (C=S), 1472 (Ar), 1524 (Ar), 1614 (Ar), $2876(\mathrm{CH}), 2938(\mathrm{CH}), 3396(\mathrm{NH})$; ${ }^{1} \mathrm{H}$ NMR $\left(\mathrm{CDCl}_{3}\right): \delta=2.38-2.42(\mathrm{~m}, 2 \mathrm{H}$, naphthyl-O$\mathrm{CH}_{2} \mathrm{CH}_{2} \mathrm{CH}_{2}-\mathrm{S}-\mathrm{C}=\mathrm{S}$ ), 2.84-2.88 (t, 2H, $\mathrm{CH}_{2}-\mathrm{S}-\mathrm{C}=\mathrm{S}$ ), 3.74 (s, $3 \mathrm{H}, \mathrm{OCH}_{3}$ ), 4.0 (bs, H, NH), 4.02-4.05 (t, 2H, $\mathrm{CH}_{2}-\mathrm{O}-$ naphthyl), 6.34-7.65 (m, 11H, Ar-H of naphthyloxy and phenyl ring); Mass: m/e 383; Analysis: $\mathrm{C}_{21} \mathrm{H}_{21} \mathrm{NO}_{2} \mathrm{~S}_{2}$, Calcd. (\%): $\mathrm{C}$, 65.76, H 5.52, N, 3.65, Obsd. (\%): C, 65.27, H, 5.85, N, 3.81.

(4-Methoxy-4-phenyl)dithiocarbamic acid-4-(naphthalen2-yloxy)butyl ester (33): Yield: 0.83 g (94.5 \%); m.p.: 126 ${ }^{\circ} \mathrm{C}$; IR (KBr, $\left.v_{\max }, \mathrm{cm}^{-1}\right)$ : $681(\mathrm{C}-\mathrm{S}), 1126(\mathrm{C}=\mathrm{S}), 1484(\mathrm{Ar})$, $1523(\mathrm{Ar}), 1610(\mathrm{Ar}), 2885(\mathrm{CH}), 2936(\mathrm{CH}), 3407(\mathrm{NH}) ;{ }^{1} \mathrm{H}$ NMR $\left(\mathrm{CDCl}_{3}\right): \delta=1.71-1.74\left(\mathrm{~m}, 2 \mathrm{H}\right.$, naphthyl-O- $\left.\mathrm{CH}_{2} \mathrm{CH}_{2}\right)$, 1.94-1.96 (m, 2H, S- $\mathrm{CH}_{2} \mathrm{CH}_{2}$ ), 2.01 (bs, H, NH), 2.82-2.86 (t, $\left.2 \mathrm{H}, \mathrm{CH}_{2}-\mathrm{S}-\mathrm{C}=\mathrm{S}\right), 3.72\left(\mathrm{~s}, 3 \mathrm{H}, \mathrm{OCH}_{3}\right), 3.92-3.94\left(\mathrm{~d}, 2 \mathrm{H}, \mathrm{CH}_{2}\right.$ of benzylic proton), 4.01-4.04 (t, 2H, $\mathrm{CH}_{2}$-O-naphthyl), 6.65$7.62(\mathrm{~m}, 11 \mathrm{H}, \mathrm{Ar}-\mathrm{H}$ of naphthyloxy and phenyl ring); Mass: m/e 411; Analysis: $\mathrm{C}_{23} \mathrm{H}_{25} \mathrm{NO}_{2} \mathrm{~S}_{2}$, Calcd. (\%): C, 67.12, H, 6.12, N, 3.40, Obsd. (\%): C, 67.67, H, 6.40, N, 3.67.

Cyclohexyl-dithiocarbamic acid-2-(naphthalen-2yloxy)ethyl ester (34): Yeild: $0.714 \mathrm{~g}(85.5 \%)$, m.p; $112{ }^{\circ} \mathrm{C}$; IR (KBr, $\left.v_{\max }, \mathrm{cm}^{-1}\right)$ : $658(\mathrm{C}-\mathrm{S}), 1103$ (C=S), $1454(\mathrm{Ar}), 1502$ (Ar), 1600 (Ar), $2851(\mathrm{CH}), 2926(\mathrm{CH}), 3373(\mathrm{NH}) ;{ }^{1} \mathrm{H}$ NMR $\left(\mathrm{CDCl}_{3}\right): \delta=1.41-1.45\left(\mathrm{~m}, 6 \mathrm{H}, \mathrm{CH}_{2}\right.$ of cyclohexyl ring), 1.62$1.64\left(\mathrm{~m}, 4 \mathrm{H}, \mathrm{CH}_{2}\right.$ of cyclohexyl ring), 2.0 (bs, $\mathrm{H}, \mathrm{NH}$ ), 2.542.58 (m, H, tertiary $\mathrm{H}$ of cyclohexyl ring), 3.26-3.29 (t, $2 \mathrm{H}$, $\mathrm{CH}_{2}-\mathrm{S}-\mathrm{C}=\mathrm{S}$ ), 4.71-4.74 (t, 2H, $\mathrm{CH}_{2}$-O-naphthyl), 6.98-7.62 (m, 7H, Ar-H of naphthyloxy); Mass: m/e 345; Analysis: $\mathrm{C}_{19} \mathrm{H}_{23} \mathrm{NOS}_{2}$, Calcd. (\%): C, 66.05, H, 6.71, N, 4.05, Obsd. (\%): C, 65.65, H, 6.97, N, 4.23.

Cyclohexyl-dithiocarbamic acid-3-(naphthalen-2yloxy)propyl ester (35): Yield: $0.714 \mathrm{~g}(85.5 \%)$; m.p.: 112 ${ }^{\circ} \mathrm{C}$; IR ( KBr, $\left.v_{\max }, \mathrm{cm}^{-1}\right): 658(\mathrm{C}-\mathrm{S}), 1103(\mathrm{C}=\mathrm{S}), 1454$ (Ar), 1502 (Ar), 1600 (Ar), $2851(\mathrm{CH}), 2927(\mathrm{CH}), 3373(\mathrm{NH}) ;{ }^{1} \mathrm{H}$ $\operatorname{NMR}\left(\mathrm{CDCl}_{3}\right): \delta=1.44-1.48$ (m, $6 \mathrm{H}, \mathrm{CH}_{2}$ of cyclohexyl ring), 1.63-1.66 (m, 4H, $\mathrm{CH}_{2}$ of cyclohexyl ring), 2.0 (bs, $\mathrm{H}, \mathrm{NH}$ ), 2.54-2.59 (m, H, tertiary $\mathrm{H}$ of cyclohexyl ring), 3.28-3.30 ( $\mathrm{t}$, $2 \mathrm{H}, \mathrm{CH}_{2}-\mathrm{S}-\mathrm{C}=\mathrm{S}$ ), 4.71-4.73 (t, 2H, $\mathrm{CH}_{2}-\mathrm{O}$-naphthyl), 6.957.62 (m, 7H, Ar-H of naphthyloxy); Mass: m/e 345; Analysis: $\mathrm{C}_{19} \mathrm{H}_{23} \mathrm{NOS}_{2}$, Calcd. (\%): C, 66.05, H, 6.71, N, 4.05, Obsd. (\%): C, 65.65, H, 6.97, N, 4.23.

Cyclohexyl-dithiocarbamic acid-4-(naphthalen-2yloxy)butyl ester (36): Yield: $0.75 \mathrm{~g}(94.5 \%)$; m.p.: $126^{\circ} \mathrm{C}$; IR (KBr, $\left.v_{\max }, \mathrm{cm}^{-1}\right): 668(\mathrm{C}-\mathrm{S}), 1121(\mathrm{C}=\mathrm{S}), 1469(\mathrm{Ar}), 1523$ (Ar), 1617 (Ar), $2879(\mathrm{CH}), 2937(\mathrm{CH}), 3408(\mathrm{NH}) ;{ }^{1} \mathrm{H}$ NMR $\left(\mathrm{CDCl}_{3}\right): \delta=1.42-1.44$ ( $\mathrm{m}, 6 \mathrm{H}, \mathrm{CH}_{2}$ of cyclohexyl ring), $1.65-$ $1.68\left(\mathrm{~m}, 4 \mathrm{H}, \mathrm{CH}_{2}\right.$ of cylohexyl ring), 1.71-1.73 (m, 2H, naphthyl-O- $\mathrm{CH}_{2} \mathrm{CH}_{2}$ ), 1.94-1.96 (m, $2 \mathrm{H}, \mathrm{S}-\mathrm{CH}_{2} \mathrm{CH}_{2}$ ), 2.0 (bs, $\mathrm{H}, \mathrm{NH}), 2.54-2.57$ (m, H, tert. $\mathrm{CH}$ of cylohexyl ring), 2.842.88 (t, 2H, $\mathrm{CH}_{2}-\mathrm{S}-\mathrm{C}=\mathrm{S}$ ), 4.02-4.06 (t, 2H, $\mathrm{CH}_{2}$-O-naphthyl), 6.98-7.62 (m, 7H, Ar-H of naphthyloxy); Mass: m/e 373; Analysis: $\mathrm{C}_{21} \mathrm{H}_{27} \mathrm{NOS}_{2}$, Calcd. (\%): C, 67.52, H, 7.28, N, 3.75, Obsd. (\%): C, 67.84, H, 7.12, N, 3.59.
Benzyl-dithiocarbamic acid-2-(naphthalen-2-yloxy)ethyl ester (37): Yield: $0.75 \mathrm{~g}(87.3 \%)$; m.p.: $101{ }^{\circ} \mathrm{C}$; $\mathrm{IR}(\mathrm{KBr}$, $\left.v_{\max }, \mathrm{cm}^{-1}\right): 662(\mathrm{C}-\mathrm{S}), 1112(\mathrm{C}=\mathrm{S}), 1464(\mathrm{Ar}), 1512(\mathrm{Ar}), 1603$ (Ar), $2865(\mathrm{CH}), 2926(\mathrm{CH}), 3385(\mathrm{NH}) ;{ }^{1} \mathrm{H} \mathrm{NMR}\left(\mathrm{CDCl}_{3}\right): \delta$ $=2.0(\mathrm{bs}, \mathrm{H}, \mathrm{NH}), 3.26-3.32\left(\mathrm{t}, 2 \mathrm{H}, \mathrm{CH}_{2}-\mathrm{S}-\mathrm{C}=\mathrm{S}\right), 3.92-3.94$ (d, 2H, benzylic proton), 4.71-4.73 (t, 2H, $\mathrm{CH}_{2}-\mathrm{O}$-naphthyl), 6.98-7.65 (m, 12H, Ar-H of naphthyloxy); Mass: m/e 353; Analysis: $\mathrm{C}_{20} \mathrm{H}_{19} \mathrm{NOS}_{2}$, Calcd. (\%): C, 67.95, H 5.42, N, 3.96, Obsd. (\%): C, 67.63, H, 5.58, N, 4.12.

Benzyl-dithiocarbamic acid-3-(naphthalen-2-yloxy)propyl ester (38): Yield: $0.75 \mathrm{~g}(89.8 \%)$; m.p.: $109^{\circ} \mathrm{C}$; IR $\left(\mathrm{KBr}, v_{\max }, \mathrm{cm}^{-1}\right)$ : $668(\mathrm{C}-\mathrm{S}), 1114(\mathrm{C}=\mathrm{S}), 1474(\mathrm{Ar}), 1514$ (Ar), 1612 (Ar), $2863(\mathrm{CH}), 2926(\mathrm{CH}), 3398(\mathrm{NH}) ;{ }^{1} \mathrm{H}$ NMR $\left(\mathrm{CDCl}_{3}\right): \delta=2.0(\mathrm{bs}, \mathrm{H}, \mathrm{NH}), 2.38-2.42(\mathrm{~m}, 2 \mathrm{H}$, naphthyl-O$\mathrm{CH}_{2} \mathrm{CH}_{2} \mathrm{CH}_{2}-\mathrm{S}-\mathrm{C}=\mathrm{S}$ ), 2.82-2.86 (t, $2 \mathrm{H}, \mathrm{CH}_{2}-\mathrm{S}-\mathrm{C}=\mathrm{S}$ ), 3.91-3.93 (d, $2 \mathrm{H}, \mathrm{CH}_{2}$ of benzylic hydrogens), 4.01-4.04 (t, 2H, $\mathrm{CH}_{2}-\mathrm{O}-$ naphthyl), 6.96-7.62 (m, 12H, Ar-H of naphthyloxy); Mass: m/e 367; Analysis: $\mathrm{C}_{21} \mathrm{H}_{21} \mathrm{NOS}_{2}$, Calcd. (\%): C, 68.63, H, 5.76, N, 3.81, Obsd. (\%): C, 68.27, H, 5.94, N, 4.02.

Benzyl-dithiocarbamic acid-4-(naphthalen-2-yloxy)butyl ester (39): Yield: $0.75 \mathrm{~g}(92.8 \%)$; m.p.: $119^{\circ} \mathrm{C}$; $\mathrm{IR}(\mathrm{KBr}$, $\left.v_{\max }, \mathrm{cm}^{-1}\right): 673(\mathrm{C}-\mathrm{S}), 1126(\mathrm{C}=\mathrm{S}), 1484(\mathrm{Ar}), 1529(\mathrm{Ar}), 1612$ (Ar), $2873(\mathrm{CH}), 2936(\mathrm{CH}), 3398(\mathrm{NH}) ;{ }^{1} \mathrm{H} \mathrm{NMR}\left(\mathrm{CDCl}_{3}\right): \delta$ $=1.71-1.74\left(\mathrm{~m}, 2 \mathrm{H}\right.$, naphthyl-O- $\left.\mathrm{CH}_{2} \mathrm{CH}_{2}\right), 1.96-1.99(\mathrm{~m}, 2 \mathrm{H}$, S- $\mathrm{CH}_{2} \mathrm{CH}_{2}$ ), 2.0 (bs, H, NH), 2.84-2.88 (t, $2 \mathrm{H}, \mathrm{CH}_{2}-\mathrm{S}-\mathrm{C}=\mathrm{S}$ ), 3.91-3.94 (d, $2 \mathrm{H}, \mathrm{CH}_{2}$ of benzylic proton), 4.01-4.04 (t, $2 \mathrm{H}$, $\mathrm{CH}_{2}$-O-naphthyl), 6.96-7.65 (m, 12H, Ar-H of naphthyloxy); Mass: m/e 381; Analysis: $\mathrm{C}_{22} \mathrm{H}_{23} \mathrm{NOS}_{2}$, Calcd. (\%): C, 69.25, H 6.08, N, 3.67, Obsd. (\%): C, 69.67, H, 5.87, N, 3.46.

Phenyl ethyl-dithiocarbamic acid-2-(naphthalen-2yloxy)ethyl ester (40): Yield: $0.78 \mathrm{~g}(88.2 \%)$; m.p.: $146^{\circ} \mathrm{C}$; IR $\left(\mathrm{KBr}, \mathrm{v}_{\max }, \mathrm{cm}^{-1}\right)$ : $661(\mathrm{C}-\mathrm{S}), 1112(\mathrm{C}=\mathrm{S}), 1464(\mathrm{Ar}), 1514$ (Ar), 1605 (Ar), $2865(\mathrm{CH}), 2923(\mathrm{CH}), 3376(\mathrm{NH}) ;{ }^{1} \mathrm{H}$ NMR $\left(\mathrm{CDCl}_{3}\right): \delta=2.0$ (bs, $\left.\mathrm{H}, \mathrm{NH}\right), 2.80-2.82\left(\mathrm{t}, 2 \mathrm{H}, \mathrm{PhCH}_{2}\right), 2.96-$ $2.98\left(\mathrm{~m}, 2 \mathrm{H}, \mathrm{NHCH}_{2} \mathrm{CH}_{2} \mathrm{Ph}\right.$ ), 3.28-3.31 (t, $2 \mathrm{H}, \mathrm{CH}_{2}-\mathrm{S}-\mathrm{C}=\mathrm{S}$ ), 4.71-4.73 (t, 2H, $\mathrm{CH}_{2}-\mathrm{O}$-naphthyl), 6.98-7.62 (m, 12H, Ar-H of naphthyloxy and phenyl ring); Mass: $\mathrm{m} / \mathrm{e}$ 367; Analysis: $\mathrm{C}_{21} \mathrm{H}_{21} \mathrm{NOS}_{2}$, Calcd. (\%): C, 68.63, H, 5.76, N, 3.81, Obsd. (\%): C, 68.19, H, 6.06, N, 3.95.

Phenyl ethyl-dithiocarbamic acid-3-(naphthalen-2yloxy)propyl ester (41): Yield: $0.8 \mathrm{~g}(91.4 \%)$; m.p.: $172^{\circ} \mathrm{C}$; IR (KBr, $\left.\nu_{\max }, \mathrm{cm}^{-1}\right)$ : $668(\mathrm{C}-\mathrm{S}), 1126(\mathrm{C}=\mathrm{S}), 1478(\mathrm{Ar}), 1519$ (Ar), 1614 (Ar), 2878 (CH), 2933 (CH), 3396 (NH); ${ }^{1} \mathrm{H}$ NMR $\left(\mathrm{CDCl}_{3}\right): \delta=2.0$ (bs, H, NH), 2.35-2.41 (m, 2H, naphthyl-O$\mathrm{CH}_{2} \mathrm{CH}_{2} \mathrm{CH}_{2}-\mathrm{S}-\mathrm{C}=\mathrm{S}$ ), 2.80-2.82 (t, $2 \mathrm{H}, \mathrm{PhCH}_{2}$ ), 2.84-2.86 (t, $2 \mathrm{H}, \mathrm{CH}_{2}-\mathrm{S}-\mathrm{C}=\mathrm{S}$ ), 2.96-3.02 (m, $\left.2 \mathrm{H}, \mathrm{CH}_{2} \mathrm{NH}\right), 4.02-4.06$ (t, $2 \mathrm{H}, \mathrm{CH}_{2}$-O-naphthyl), 6.95-7.62 (m, 12H, Ar-H of naphthyloxy and phenyl group); Mass: m/e 381; Analysis: $\mathrm{C}_{22} \mathrm{H}_{23} \mathrm{NOS}_{2}$, Calcd. (\%): C, 69.25, H, 6.08, N, 3.67, Obsd. (\%): C, 68.87, $\mathrm{H}, 6.29, \mathrm{~N}, 3.89$.

Phenyl ethyl-dithiocarbamic acid-4-(naphthalen-2yloxy)butyl ester (42): Yield: $0.8 \mathrm{~g}(94.8 \%)$; m.p.: $179^{\circ} \mathrm{C}$; IR (KBr, $\left.v_{\max }, \mathrm{cm}^{-1}\right)$ : 679 (C-S), $1149(\mathrm{C}=\mathrm{S}), 1487(\mathrm{Ar}), 1533$ (Ar), 1622 (Ar), $2884(\mathrm{CH}), 2944(\mathrm{CH}), 3438(\mathrm{NH}) ;{ }^{1} \mathrm{H}$ NMR $\left(\mathrm{CDCl}_{3}\right): \delta=1.72-1.74\left(\mathrm{~m}, 2 \mathrm{H}\right.$, naphthyl-O- $\left.\mathrm{CH}_{2} \mathrm{CH}_{2}\right), 1.96-$ 1.97 (m, 2H, S- $\mathrm{CH}_{2} \mathrm{CH}_{2}$ ), 2.01 (bs, H, NH), 2.80-2.82 (t, 2H, $\mathrm{PhCH}_{2}$ ), 2.86-2.88 (t, 2H, $\left.\mathrm{CH}_{2}-\mathrm{S}-\mathrm{C}=\mathrm{S}\right), 2.96-3.00(\mathrm{~m}, 2 \mathrm{H}$, $\mathrm{CH}_{2} \mathrm{NH}$ ), 4.02-4.06 (t, 2H, $\mathrm{CH}_{2}$-O-naphthyl), 6.98-7.62 (m, 
$12 \mathrm{H}, \mathrm{Ar}-\mathrm{H}$ of naphthyloxy and phenyl ring); Mass: $m / e$ 395; Analysis: $\mathrm{C}_{23} \mathrm{H}_{25} \mathrm{NOS}_{2}$, Calcd. (\%): C, 69.93, H, 6.37, N, 3.54, Obsd. (\%): C, 69.57, H, 6.55, N, 3.72.

Phenyl propyl-dithiocarbamic acid-2-(naphthalen-2yloxy)ethyl ester (43): Yield: $0.84 \mathrm{~g}(90.2 \%)$; m.p.: $119{ }^{\circ} \mathrm{C}$; IR (KBr, $\left.\nu_{\max }, \mathrm{cm}^{-1}\right)$ : $668(\mathrm{C}-\mathrm{S}), 1114(\mathrm{C}=\mathrm{S}), 1462(\mathrm{Ar}), 1514$ (Ar), 1600 (Ar), $2862(\mathrm{CH}), 2925(\mathrm{CH}), 3388(\mathrm{NH}) ;{ }^{1} \mathrm{H}$ NMR $\left(\mathrm{CDCl}_{3}\right): \delta=1.86-1.88\left(\mathrm{~m}, 2 \mathrm{H}, \mathrm{PhCH}_{2} \mathrm{CH}_{2} \mathrm{CH}_{2} \mathrm{NH}\right), 2.0$ (bs, $\mathrm{H}, \mathrm{NH}), 2.54-2.56\left(\mathrm{t}, 2 \mathrm{H}, \mathrm{PhCH}_{2}\right), 2.65-2.64(\mathrm{~m}, 2 \mathrm{H}$, $\mathrm{NHCH}_{2} \mathrm{CH}_{2} \mathrm{CH}_{2} \mathrm{Ph}$ ), 3.28-3.31 (t, 2H, $\mathrm{CH}_{2}-\mathrm{S}-\mathrm{C}=\mathrm{S}$ ), 4.72-4.74 (t, 2H, $\mathrm{CH}_{2}$-O-naphthyl), 6.95-7.62 (m, 12H, Ar-H of naphthyloxy and phenyl ring); Mass: $\mathrm{m} / \mathrm{e}$ 381; Analysis: $\mathrm{C}_{22} \mathrm{H}_{23} \mathrm{NOS}_{2}$, Calcd. (\%): C, 69.25, H 6.08, N, 3.67, Obsd. (\%): C, 69.66, H, 5.99, N, 3.35.

Phenyl propyl-dithiocarbamic acid-3-(naphthalen-2yloxy)propyl ester (44): Yield: $0.84 \mathrm{~g}$ (93.2 \%); m.p.: 135 ${ }^{\circ} \mathrm{C}$; IR (KBr, $\left.v_{\max }, \mathrm{cm}^{-}{ }^{-}\right): 682(\mathrm{C}-\mathrm{S}), 1129(\mathrm{C}=\mathrm{S}), 1481$ (Ar), 1533 (Ar), 1626 (Ar), $2884(\mathrm{CH}), 2936(\mathrm{CH}), 3416(\mathrm{NH}) ;{ }^{1} \mathrm{H}$ NMR $\left(\mathrm{CDCl}_{3}\right): \delta=1.86-1.89$ (m, $\left.2 \mathrm{H}, \mathrm{Ph}^{-} \mathrm{CH}_{2} \mathrm{CH}_{2} \mathrm{CH}_{2}\right), 2.0$ (bs, $\mathrm{H}, \mathrm{NH}), 2.38-2.42$ (m, $2 \mathrm{H}$, naphthyl-O- $\mathrm{CH}_{2} \mathrm{CH}_{2} \mathrm{CH}_{2}-\mathrm{S}-$ $\mathrm{C}=\mathrm{S}), 2.52-2.55$ (t, $2 \mathrm{H}, \mathrm{PhCH}_{2}$ ), 2.62-2.64 (m, 2H, $\mathrm{NHCH}_{2}$ ), 2.84-2.88 (t, 2H, $\left.\mathrm{CH}_{2}-\mathrm{S}-\mathrm{C}=\mathrm{S}\right), 4.02-4.05$ (t, $2 \mathrm{H}, \mathrm{CH}_{2}-\mathrm{O}-$ naphthyl), 6.98-7.65 (m, 12H, Ar-H of naphthyloxy and phenyl group); Mass: m/e 395; Analysis: $\mathrm{C}_{23} \mathrm{H}_{25} \mathrm{NOS}_{2}$, Calcd. (\%): C, 69.83, H 6.37, N, 3.54, Obsd. (\%): C, 69.34, H, 6.66, N, 3.74.

Phenyl propyl-dithiocarbamic acid-4-(naphthalen-2yloxy)butyl ester (45): Yield: $0.85 \mathrm{~g}(97.6 \%)$; m.p.: $154{ }^{\circ} \mathrm{C}$; IR $\left(\mathrm{KBr}, \mathrm{v}_{\max }, \mathrm{cm}^{-1}\right)$ : $692(\mathrm{C}-\mathrm{S}), 1139(\mathrm{C}=\mathrm{S}), 1486(\mathrm{Ar}), 1539$ (Ar), 1628 (Ar), $2882(\mathrm{CH}), 2948(\mathrm{CH}), 3427(\mathrm{NH}) ;{ }^{1} \mathrm{H}$ NMR $\left(\mathrm{CDCl}_{3}\right): \delta=1.71-1.73\left(\mathrm{~m}, 2 \mathrm{H}\right.$, naphthyl-O- $\left.\mathrm{CH}_{2} \mathrm{CH}_{2}\right), 1.86-$ $1.88\left(\mathrm{~m}, 2 \mathrm{H}, \mathrm{PhCH}_{2} \mathrm{CH}_{2} \mathrm{CH}_{2} \mathrm{NH}\right)$, 1.96-1.99 (m, 2H, S$\mathrm{CH}_{2} \mathrm{CH}_{2}$ ), 2.02 (bs, $\mathrm{H}, \mathrm{NH}$ ), 2.54-2.56 (t, $2 \mathrm{H}, \mathrm{PhCH}_{2}$ ), 2.64$2.68\left(\mathrm{~m}, 2 \mathrm{H}, \mathrm{PhCH}_{2} \mathrm{CH}_{2} \mathrm{CH}_{2}-\mathrm{N}\right), 2.84-2.86$ (t, $\left.2 \mathrm{H}, \mathrm{CH}_{2}-\mathrm{S}-\mathrm{C}=\mathrm{S}\right)$, 2.98-3.01 (m, $\left.2 \mathrm{H}, \mathrm{CH}_{2} \mathrm{NH}\right), 4.02-4.06$ (t, $2 \mathrm{H}, \mathrm{CH}_{2}-\mathrm{O}-$ naphthyl), 6.98-7.62 (m, 12H, Ar-H of naphthyloxy and phenyl ring); Mass: m/e 409; Analysis: $\mathrm{C}_{24} \mathrm{H}_{27} \mathrm{NOS}_{2}$, Calcd. (\%): $\mathrm{C}$, 70.37, H, 6.64, N, 3.42, Obsd. (\%): C, 69.95, H, 6.86, N, 3.62.

Di-sec-butyl-dithiocarbamic acid-2-(naphthalen-2yloxy)ethyl ester (46): Yield: $0.84 \mathrm{~g}(90.2 \%)$; m.p.: $119^{\circ} \mathrm{C}$; IR $\left(\mathrm{KBr}, v_{\max }, \mathrm{cm}^{-1}\right)$ : 668 (C-S), $1115(\mathrm{C}=\mathrm{S}), 1462(\mathrm{Ar}), 1514(\mathrm{Ar})$, $1601(\mathrm{Ar}), 2865(\mathrm{CH}), 2926(\mathrm{CH}), 3388(\mathrm{NH}) ;{ }^{1} \mathrm{HNMR}\left(\mathrm{CDCl}_{3}\right)$ : $\delta=1.08-1.10\left(\mathrm{~d}, \mathrm{CH}_{3}\right), 2.75-2.79\left(\mathrm{~m}, 8 \mathrm{H}, \mathrm{CH}_{3} \mathrm{CH}_{2} \mathrm{CH}_{3}\right), 1.38-$ $1.41\left(\mathrm{M}, 6 \mathrm{H}, \mathrm{CH}_{3} \mathrm{CHCH}_{3}\right), 0.94-0.96\left(\mathrm{t}, 2 \mathrm{H}, \mathrm{CH}_{3} \mathrm{CHCH}_{2}\right), 3.28-$ 3.30 (t, 2H, $\left.\mathrm{CH}_{2}-\mathrm{S}-\mathrm{C}=\mathrm{S}\right), 4.71-4.73$ (t, 2H, $\mathrm{CH}_{2}-\mathrm{O}$-naphthyl), 6.95-7.63 (m, 12H, Ar-H of naphthyloxy and phenyl ring); Mass: m/e 375.59; Analysis: $\mathrm{C}_{21} \mathrm{H}_{29} \mathrm{NOS}_{2}$, Calcd. (\%): C, 67.10, H 7.75, N, 3.70, O, 4.22, S, 17.05. Obsd. (\%): C, 67.15, H, 7.78, N, 3.73, O, 4.26, S, 17.07.

Di-sec-butyl-dithiocarbamic acid-3-(naphthalen-2yloxy)propyl ester (47): Yield: $0.84 \mathrm{~g}(90.2 \%)$; m.p.: 119 ${ }^{\circ} \mathrm{C}$; IR (KBr, $\left.v_{\max }, \mathrm{cm}^{-1}\right)$ : $668(\mathrm{C}-\mathrm{S}), 1114(\mathrm{C}=\mathrm{S}), 1462(\mathrm{Ar})$, 1514 (Ar), 1600 (Ar), 2862 (CH), $2925(\mathrm{CH}), 3388(\mathrm{NH}) ;{ }^{1} \mathrm{H}$ NMR $\left(\mathrm{CDCl}_{3}\right): \delta=1.08-1.10\left(\mathrm{~d}, \mathrm{CH}_{3}\right), 2.75-2.79(\mathrm{~m}, 8 \mathrm{H}$, $\mathrm{CH}_{3} \mathrm{CH}_{2} \mathrm{CH}_{3}$ ), 1.38-1.41 (M, 6H, $\mathrm{CH}_{3} \mathrm{CHCH}_{3}$ ), 0.94-0.96 (t, $2 \mathrm{H}, \mathrm{CH}_{3} \mathrm{CHCH}_{2}$ ), 3.28-3.30 (t, 2H, $\mathrm{CH}_{2}-\mathrm{S}-\mathrm{C}=\mathrm{S}$ ), 2.35-2.39(m, $2 \mathrm{H}, \mathrm{CH}_{2} \mathrm{CH}_{2} \mathrm{CH}_{2}$ ), 4.72-4.74 (t, 2H, $\mathrm{CH}_{2}$-O-naphthyl), 6.967.62 (m, 12H, Ar-H of naphthyloxy and phenyl ring); Mass: m/e 389.62; Analysis: $\mathrm{C}_{22} \mathrm{H}_{31} \mathrm{NOS}_{2}$, Calcd. (\%): C, 67.80, H
8.01, N, 3.60, O, 4. 10, S, 16.42. Obsd. (\%): C, 67.82, H, 8.02, $\mathrm{N}, 3.59, \mathrm{O}, 4.11, \mathrm{~S}, 16.46$.

Di-sec-butyl-dithiocarbamic acid-4-(naphthalen-2yloxy)butyl ester (48): Yield: $0.84 \mathrm{~g}(90.2 \%)$; m.p.: $119^{\circ} \mathrm{C}$; IR $\left(\mathrm{KBr}, v_{\max }, \mathrm{cm}^{-1}\right)$ : $668(\mathrm{C}-\mathrm{S}), 1114(\mathrm{C}=\mathrm{S}), 1465$ (Ar), $1514(\mathrm{Ar})$, 1600 (Ar), $2865(\mathrm{CH}), 2927(\mathrm{CH}), 3388(\mathrm{NH}) ;{ }^{1} \mathrm{H} \mathrm{NMR}\left(\mathrm{CDCl}_{3}\right)$ : $\delta=1.08-1.10\left(\mathrm{~d}, \mathrm{CH}_{3}\right), 2.75-2.79\left(\mathrm{~m}, 8 \mathrm{H}, \mathrm{CH}_{3} \mathrm{CH}_{2} \mathrm{CH}_{3}\right), 1.38$ $1.41\left(\mathrm{M}, 6 \mathrm{H}, \mathrm{CH}_{3} \mathrm{CHCH}_{3}\right), 0.94-0.96\left(\mathrm{t}, 2 \mathrm{H}, \mathrm{CH}_{3} \mathrm{CHCH}_{2}\right), 3.27-$ 3.31 (t, 2H, CH$\left.{ }_{2}-\mathrm{S}-\mathrm{C}=\mathrm{S}\right), 1.92-1.96\left(\mathrm{~m}, 2 \mathrm{H}, \mathrm{CH}_{2} \mathrm{CH}_{2} \mathrm{CH}_{2}\right.$ ), 1.68-1.71 (m, 2H, $\mathrm{CH}_{2} \mathrm{CH}_{2} \mathrm{CH}_{2}$ ), 4.71-4.73 (t, $2 \mathrm{H}, \mathrm{CH}_{2}-\mathrm{O}-$ naphthyl), 6.97-7.64 (m, 12H, Ar-H of naphthyloxy and phenyl ring); Mass: m/e 403.64; Analysis: $\mathrm{C}_{23} \mathrm{H}_{33} \mathrm{NOS}_{2}$, Calcd. (\%): C, 68.41, H 8.20, N, 3.44, O, 3.92, S, 15.87. Obsd. (\%): C, 68.44, H, 8.24, N, 3.47, O, 3.96, S, 15.89.

\section{RESULTS AND DISCUSSION}

The synthetic route of $\omega$-substituted 2-naphthyloxy haloalkanes and desired products (4-48) as shown in Scheme-I is prepared by direct condensation of $\beta$-naphthol and alkyl dihalide. Intermediate $\omega$-substituted 2-naphthyloxy haloalkanes (3) was prepared by reacting alkyl dihalide (2) with $\beta$-naphthol (1) in the presence of anhydrous $\mathrm{K}_{2} \mathrm{CO}_{3}$ which was subsequently converted to corresponding dithiocarbamates of $\mathbf{4}$ to $\mathbf{4 8}$ by reaction of various types of primary and secondary amines involving Triton $\mathrm{B} / \mathrm{CS}_{2}$ system at room temperature. Hence, dithiocarbamates of desired (4-48) series is synthesized employing various kinds of aliphatic, aromatic, alicyclic, heterocyclic primary and secondary amines. The protocol proved to be successful and the desired product was isolated and further confirmed by spectroscopic and analytical methods.

The structural characterization of title compounds have been done by melting point, ${ }^{1} \mathrm{H}$ NMR, ${ }^{13} \mathrm{C}$ NMR and highresolution mass spectrometry (HRMS). All spectral data were consistent with the assigned structures. The comparative study of the yield of $\mathbf{3 a}, \mathbf{3 b}$ and $\mathbf{3 c}$ increases with number of carbon due to $+\mathrm{I}$ effect. However, final yield of dithiocarbamates of Prototype I (4-48) is dependent upon the electron releasing effect of the amines like pyrrolidine, piperidine, $N$-methyl piperazine, cyclohexane, phenyl ethyl amine and phenyl propyl amine show higher yield compared to primary amines (Table-1).

Antimicrobial screening: The series of compounds were screened for antimicrobial activity through microdilution method using various bacterial and fungal strains. The antifungal and antibacteriaal values were estimated as MIC values. Fluconazole and ciprofloxacin were used as the standard antifungal and antibacterial drug. As shown in Table-2, the SAR of these compounds can be studied by variying the alkyl chain and amines attached to these range of compounds. Compounds having three-carbon chain attached to them are found to be more active as compared to two-carbon or four-carbon chain. Among 28, 29, 30 and 31, 32, 33, 29 and 33 were found to possess higher potency as compared to others because of the three-carbon chain attached to them. The higher potency of three-carbon chain is due to hydrophilicity. Upon studying the effect of various types of amines, we found that compounds like 28, 29, 30, 31, 32 and 33 having aromatic amine like anisidine and toludine possessed comparable values to control drugs. Substitution of heterocyclic amines in compounds 16, 
TABLE-1

SYNTHESIS OF VARIOUS TYPES OF DITHIOCARBAMATES OF $\omega$-SUBSTITUTED 2-NAPHTHYLOXY ALKANES

\begin{tabular}{|c|c|c|c|c|c|}
\hline Comp. No. & $\mathrm{n}$ & $\mathrm{R}_{1}$ & $\mathrm{R}_{2}$ & Time $(\min )$ & Yield $(\%)$ \\
\hline 4 & 1 & $\mathrm{C}_{4} \mathrm{H}_{9}$ & $\mathrm{H}$ & 35 & 93 \\
\hline 5 & 2 & $\mathrm{C}_{4} \mathrm{H}_{9}$ & $\mathrm{H}$ & 30 & 95 \\
\hline 6 & 3 & $\mathrm{C}_{4} \mathrm{H}_{9}$ & $\mathrm{H}$ & 38 & 93 \\
\hline 7 & 1 & $\mathrm{C}_{6} \mathrm{H}_{11}$ & $\mathrm{H}$ & 30 & 94 \\
\hline 8 & 2 & $\mathrm{C}_{6} \mathrm{H}_{11}$ & $\mathrm{H}$ & 30 & 95 \\
\hline 9 & 3 & $\mathrm{C}_{6} \mathrm{H}_{11}$ & $\mathrm{H}$ & 35 & 94 \\
\hline 10 & 1 & $\mathrm{C}_{8} \mathrm{H}_{15}$ & $\mathrm{H}$ & 40 & 90 \\
\hline 11 & 2 & $\mathrm{C}_{8} \mathrm{H}_{15}$ & $\mathrm{H}$ & 35 & 94 \\
\hline 12 & 3 & $\mathrm{C}_{8} \mathrm{H}_{15}$ & $\mathrm{H}$ & 38 & 92 \\
\hline 13 & 1 & $\mathrm{C}_{10} \mathrm{H}_{19}$ & $\mathrm{H}$ & 40 & 93 \\
\hline 14 & 2 & $\mathrm{C}_{10} \mathrm{H}_{19}$ & $\mathrm{H}$ & 38 & 95 \\
\hline 15 & 3 & $\mathrm{C}_{10} \mathrm{H}_{19}$ & $\mathrm{H}$ & 40 & 92 \\
\hline 16 & 1 & $\mathrm{R}_{1}=\mathrm{R}_{2}=$ Pyrrolidine & - & 25 & 96 \\
\hline 17 & 2 & $\mathrm{R}_{1}=\mathrm{R}_{2}=$ Pyrrolidine & - & 20 & 98 \\
\hline 18 & 3 & $\mathrm{R}_{1}=\mathrm{R}_{2}=$ Pyrrolidine & - & 25 & 95 \\
\hline 19 & 1 & $\mathrm{R}_{1}=\mathrm{R}_{2}=$ Piperidine & - & 30 & 98 \\
\hline 20 & 2 & $\mathrm{R}_{1}=\mathrm{R}_{2}=$ Piperidine & - & 28 & 95 \\
\hline 21 & 3 & $\mathrm{R}_{1}=\mathrm{R}_{2}=$ Piperidine & - & 30 & 98 \\
\hline 22 & 1 & $\mathrm{R}_{1}=\mathrm{R}_{2}=N$-methyl piperazine & - & 20 & 98 \\
\hline 23 & 2 & $\mathrm{R}_{1}=\mathrm{R}_{2}=N$-methyl piperazine & - & 20 & 98 \\
\hline 24 & 3 & $\mathrm{R}_{1}=\mathrm{R}_{2}=N$-methyl piperazine & - & 20 & 96 \\
\hline 25 & 1 & $\mathrm{R}_{1}=\mathrm{R}_{2}=$ Morpholine & - & 25 & 90 \\
\hline 26 & 2 & $\mathrm{R}_{1}=\mathrm{R}_{2}=$ Morpholine & - & 20 & 92 \\
\hline 27 & 3 & $\mathrm{R}_{1}=\mathrm{R}_{2}=$ Morpholine & - & 25 & 90 \\
\hline 28 & 1 & $\mathrm{R}_{1}=\mathrm{R}_{2}=$ Toludine & - & 25 & 92 \\
\hline 29 & 2 & $\mathrm{R}_{1}=\mathrm{R}_{2}=$ Toludine & - & 20 & 94 \\
\hline 30 & 3 & $\mathrm{R}_{1}=\mathrm{R}_{2}=$ Toludine & - & 25 & 90 \\
\hline 31 & 1 & $\mathrm{R}_{1}=\mathrm{R}_{2}=$ Anisidine & - & 30 & 92 \\
\hline 32 & 2 & $\mathrm{R}_{1}=\mathrm{R}_{2}=$ Anisidine & - & 20 & 90 \\
\hline 33 & 3 & $\mathrm{R}_{1}=\mathrm{R}_{2}=$ Anisidine & - & 25 & 92 \\
\hline 34 & 1 & $\mathrm{R}_{1}=\mathrm{R}_{2}=$ Cyclohexane & - & 25 & 95 \\
\hline 35 & 2 & $\mathrm{R}_{1}=\mathrm{R}_{2}=$ Cyclohexane & - & 25 & 92 \\
\hline 36 & 3 & $\mathrm{R}_{1}=\mathrm{R}_{2}=$ Cyclohexane & - & 30 & 94 \\
\hline 37 & 1 & $\mathrm{Ph}\left(\mathrm{CH}_{2}\right)$ & $\mathrm{H}$ & 40 & 93 \\
\hline 38 & 2 & $\mathrm{Ph}\left(\mathrm{CH}_{2}\right)$ & $\mathrm{H}$ & 25 & 96 \\
\hline 39 & 3 & $\mathrm{Ph}\left(\mathrm{CH}_{2}\right)$ & $\mathrm{H}$ & 30 & 98 \\
\hline 40 & 1 & $\mathrm{Ph}\left(\mathrm{CH}_{2} \mathrm{CH}_{2}\right)$ & $\mathrm{H}$ & 20 & 98 \\
\hline 41 & 2 & $\mathrm{Ph}\left(\mathrm{CH}_{2} \mathrm{CH}_{2}\right)$ & $\mathrm{H}$ & 20 & 98 \\
\hline 42 & 3 & $\mathrm{Ph}\left(\mathrm{CH}_{2} \mathrm{CH}_{2}\right)$ & $\mathrm{H}$ & 25 & 90 \\
\hline 43 & 1 & $\mathrm{Ph}\left(\mathrm{CH}_{2} \mathrm{CH}_{2} \mathrm{CH}_{2}\right)$ & $\mathrm{H}$ & 25 & 92 \\
\hline 44 & 2 & $\mathrm{Ph}\left(\mathrm{CH}_{2} \mathrm{CH}_{2} \mathrm{CH}_{2}\right)$ & $\mathrm{H}$ & 30 & 92 \\
\hline 45 & 3 & $\mathrm{Ph}\left(\mathrm{CH}_{2} \mathrm{CH}_{2} \mathrm{CH}_{2}\right)$ & $\mathrm{H}$ & 25 & 95 \\
\hline 46 & 1 & $\mathrm{R}_{1}=\mathrm{R}_{2}=$ Dibutyl & - & 30 & 97 \\
\hline 47 & 2 & $\mathrm{R}_{1}=\mathrm{R}_{2}=$ Dibutyl & - & 25 & 95 \\
\hline 48 & 3 & $\mathrm{R}_{1}=\mathrm{R}_{2}=$ Dibutyl & - & 35 & 80 \\
\hline
\end{tabular}

$17,18,19,20,21,22,23,24,25,26$ and 27 gave promising result. Among substituted aromatic amines benzyl amine (37, 38 and 39) gave better result than phenyl ethyl $(\mathbf{4 0}, \mathbf{4 1}$ and $\mathbf{4 2})$ and phenyl propyl amine $(\mathbf{4 3}, 44$ and $\mathbf{4 5})$.

\section{Conclusion}

In conclusion, a convenient and efficient protocol for onepot synthesis has been developed, employing three components coupling of various amines with variety of via $\mathrm{CS}_{2}$ Bridge using Triton-B. This method produces the corresponding dithiocarbamates in good to excellent yields. Furthermore, the compounds produces by this method exhibited maximum potency for antifungal and antibacterial activity which is comparable to standard drug.

\section{ACKNOWLEDGEMENTS}

The authors gratefully acknowledge fruitful discussions with Dr. Devdutt Chaturvedi and Amity University Uttar Pradesh (AUUP), Lucknow Campus, Lucknow, U.P., for their constant encouragement and support for research. Financial support from the Department of Science and Technology (DST), Govt. of India (Grant No.SR/FT/CS-147/2010) is gratefully acknowledged.

\section{CONFLICT OF INTEREST}

The authors declare that there is no conflict of interests regarding the publication of this article. 


\begin{tabular}{|c|c|c|c|c|c|c|c|c|}
\hline \multirow{3}{*}{ Compounds } & \multicolumn{8}{|c|}{$\begin{array}{c}\text { TABLE-2 } \\
\text { ANTIMICROBIAL DATA OF DITHIOCARBAMATES OF } \omega \text {-SUBSTITUTED 2-NAPHTHYLOXY ALKANES }\end{array}$} \\
\hline & \multicolumn{4}{|c|}{ Antifungal activity } & \multicolumn{4}{|c|}{ Antibacterial activity } \\
\hline & C. albicans & S. schenckii & A. fumigatus & A. niger & S. aureus & MRSA $^{*}$ & E. coli & P. aeruginosa \\
\hline 4 & 1.56 & 1.52 & 6.25 & 8 & 6.25 & $>50$ & 3.12 & 6.25 \\
\hline 5 & 1.58 & 1.56 & 6.27 & 8.5 & 6.27 & $>50$ & 3.14 & 6.26 \\
\hline 6 & 1.60 & 1.58 & 6.28 & 9 & 6.28 & $>50$ & 3.18 & 6.28 \\
\hline 7 & 3.12 & 1.56 & $>50$ & 8 & 8 & $>50$ & 6.25 & $>50$ \\
\hline 8 & 3.14 & 1.58 & $>50$ & 8.5 & 8.8 & $>50$ & 6.27 & $>50$ \\
\hline 9 & 3.18 & 1.62 & $>50$ & 8.8 & 9 & $>50$ & 6.28 & $>50$ \\
\hline 10 & 6.25 & 6.25 & $>50$ & 16 & 16 & $>50$ & $>50$ & $>50$ \\
\hline 11 & 6.27 & 6.27 & $>50$ & 15 & 17 & $>50$ & $>50$ & $>50$ \\
\hline 12 & 6.30 & 6.28 & $>50$ & 18 & 18 & $>50$ & $>50$ & $>50$ \\
\hline 13 & 12.5 & 6.25 & $>50$ & $>50$ & $>50$ & $>50$ & $>50$ & $>50$ \\
\hline 14 & 12.8 & 6.28 & $>50$ & $>50$ & $>50$ & $>50$ & $>50$ & $>50$ \\
\hline 15 & 13.0 & 6.28 & $>50$ & $>50$ & $>50$ & $>50$ & $>50$ & $>50$ \\
\hline 16 & 0.92 & 0.32 & 0.80 & 1.30 & 3.12 & $>50$ & 0.62 & 3.12 \\
\hline 17 & 0.75 & 0.28 & 0.71 & 1.20 & 3.14 & $>50$ & 0.48 & 3.14 \\
\hline 18 & 0.82 & 0.32 & 0.78 & 1.25 & 3.18 & $>50$ & 0.54 & 3.16 \\
\hline 19 & 0.95 & 0.35 & 0.89 & 25 & 6.25 & $>50$ & 0.84 & 6.25 \\
\hline 20 & 0.80 & 0.30 & 0.78 & 24 & 6.27 & $>50$ & 0.55 & 6.26 \\
\hline 21 & 0.88 & 0.33 & 0.85 & 28 & 6.28 & $>50$ & 0.72 & 6.28 \\
\hline 22 & 0.88 & 0.30 & 0.75 & 0.82 & 0.78 & 50 & 0.42 & 1.38 \\
\hline 23 & 0.70 & 0.26 & 0.66 & 0.64 & 0.64 & 50 & 0.24 & 1.33 \\
\hline 24 & 0.80 & 0.28 & 0.70 & 0.74 & 0.70 & 50 & 0.36 & 1.35 \\
\hline 25 & 0.81 & 0.29 & 0.68 & 0.66 & 0.76 & 30 & 0.32 & 1.25 \\
\hline 26 & 0.65 & 0.20 & 0.59 & 0.52 & 0.60 & 24 & 0.20 & 1.27 \\
\hline 27 & 0.74 & 0.25 & 0.62 & 0.58 & 0.68 & 28 & 0.25 & 1.29 \\
\hline 28 & 0.80 & 0.25 & 0.62 & 0.58 & 0.68 & 27 & 0.30 & 0.72 \\
\hline 29 & 0.64 & 0.17 & 0.55 & 0.45 & 0.57 & 20 & 0.15 & 0.52 \\
\hline 30 & 0.72 & 0.20 & 0.58 & 0.50 & 0.61 & 25 & 0.19 & 0.65 \\
\hline 31 & 0.78 & 0.20 & 0.52 & 0.50 & 0.59 & 22 & 0.20 & 0.68 \\
\hline 32 & 0.50 & 0.15 & 0.45 & 0.40 & 0.45 & 18 & 0.10 & 0.48 \\
\hline 33 & 0.65 & 0.18 & 0.50 & 0.45 & 0.55 & 20 & 0.15 & 0.55 \\
\hline 34 & 2.89 & 3.12 & $>50$ & $>50$ & 8 & $>50$ & 8 & 12.5 \\
\hline 35 & 2.90 & 3.14 & $>50$ & $>50$ & 8.5 & $>50$ & 8.5 & 12.7 \\
\hline 36 & 3.28 & 3.20 & $>50$ & $>50$ & 9 & $>50$ & 9 & 13.0 \\
\hline 37 & 1.05 & $>50$ & 50 & $>50$ & $>50$ & $>50$ & 50 & 8 \\
\hline 38 & 0.95 & $>50$ & 50 & $>50$ & $>50$ & $>50$ & 50 & 8.8 \\
\hline 39 & 1.10 & $>50$ & 50 & $>50$ & $>50$ & $>50$ & 50 & 9 \\
\hline 40 & 1.16 & $>50$ & $>50$ & $>50$ & $>50$ & $>50$ & $>50$ & $>50$ \\
\hline 41 & 1.05 & $>50$ & $>50$ & $>50$ & $>50$ & $>50$ & $>50$ & $>50$ \\
\hline 42 & 1.22 & $>50$ & $>50$ & $>50$ & $>50$ & $>50$ & $>50$ & $>50$ \\
\hline 43 & $>50$ & $>50$ & $>50$ & $>50$ & $>50$ & $>50$ & $>50$ & $>50$ \\
\hline 44 & $>50$ & $>50$ & $>50$ & $>50$ & $>50$ & $>50$ & $>50$ & $>50$ \\
\hline 45 & $>50$ & $>50$ & $>50$ & $>50$ & $>50$ & $>50$ & $>50$ & $>50$ \\
\hline 46 & $>50$ & $>50$ & $>50$ & $>50$ & $>50$ & $>50$ & $>50$ & $>50$ \\
\hline 47 & $>50$ & $>50$ & $>50$ & $>50$ & $>50$ & $>50$ & $>50$ & $>50$ \\
\hline 48 & $>50$ & $>50$ & $>50$ & $>50$ & $>50$ & $>50$ & $>50$ & $>50$ \\
\hline Fluconazone & 0.25 & 0.09 & 0.35 & 0.25 & - & - & - & - \\
\hline Ciprofloxacin & - & - & - & - & 0.25 & 16 & 0.03 & 0.25 \\
\hline
\end{tabular}

\section{REFERENCES}

1. S.J. Shaw, Mini Rev. Med. Chem., 8, 276 (2008); https://doi.org/10.2174/138955708783744137.

2. D.K. Hutchinson, Curr. Top. Med. Chem., 3, 1021 (2003); https://doi.org/10.2174/1568026033452195.

3. T. Asaka, A. Manaka and H. Sugiyama, Curr. Top. Med. Chem., 3, 961 (2003); https://doi.org/10.2174/1568026033452140.

4. D. Sze, K. Miller and B. Neilan, Recent Patents Anticancer. Drug Discov., 3, 14 (2008); https://doi.org/10.2174/157489208783478685.

5. D.J. Halls, Mikrochim. Acta, 57, 62 (1969); https://doi.org/10.1007/BF01216666.
6. T.W. Greene and T.P.G.M. Wuts, Protective Group in Organic Synthesis. John Wiley \& Sons, Inc.: New York, edn 4 (2007);

7. P.J. Kociensiki, Protective Groups, Thieme-Verlag, Stuttgart, edn 3 (2003).

8. J.P. Mayer, G.S. Lewis, M.J. Curtis and J. Zhang, Tetrahedron Lett., 38, 8445 (1997) https://doi.org/10.1016/S0040-4039(97)10276-3.

9. H.P. Buchstaller, Tetrahedron, 54, 3465 (1998); https://doi.org/10.1016/S0040-4020(98)00079-9.

10. P.T. Holte, L. Thijs and B. Zwanenburg, Tetrahedron Lett., 39, 7404 (1998); https://doi.org/10.1016/S0040-4039(98)01608-6.

11. Y. Liu, K. Cui, W. Lu, W. Luo, J. Wang, J. Huang and C. Guo, Molecules, 16, 4527 (2011);

https://doi.org/10.3390/molecules 16064527. 
12. F. Ozkanli, A.G. Ozkanli, K. Ozadali, E. Yildirim and K. Erol, FABAD J. Pharm. Sci., 35, 19 (2010).

13. L. Yurttas, Y. Özkay, M. Duran, G. Turan-Zitouni, A. Özdemir, Z. Cantürk, K. Küçükoglu and Z.A. Kaplancikli, Phosphorus Sulphur Silicon Rel. Elem., 191, 1166 (2016); https://doi.org/10.1080/10426507.2016.1150277.

14. K.M. Heda and S.P. Deshmukh, Int. J. Chem. Sci., 9, 1705 (2011).

15. K. Bacharaju, S.R. Jambula, S. Sivan, S.J. Tangeda and V. Manga, Bioorg. Med. Chem. Lett., 22, 3274 (2012); https://doi.org/10.1016/j.bmcl.2012.03.018.

16. Y. Horita, T. Takii, R. Kuroishi, T. Chiba, K. Ogawa, L. Kremer, Y. Sato, Y.S. Lee, T. Hasegawa and K. Onozaki, Bioorg. Med. Chem., 21, 899 (2011) https://doi.org/10.1016/j.bmcl.2010.12.084.

17. Y. Zou, S. Yu, R. Li, Q. Zhao, X. Li, M. Wu, T. Huang, X. Chai, H. Hu and Q. Wu, Eur. J. Med. Chem., 74, 366 (2014); https://doi.org/10.1016/j.ejmech.2014.01.009.

18. S.L. Cao, Y. Wang, L. Zhu, J. Liao, Y.W. Guo, L.L. Chen, H.Q. Liu and X. Xu, Eur. J. Med. Chem., 45, 3850 (2010); https://doi.org/10.1016/i.eimech.2010.05.038

19. M.A.-H. Zahran, T.A.-R. Salem, R.M. Samaka, H.S. Agwa and A.R. Awad, Bioorg. Med. Chem., 16, 9708 (2008); https://doi.org/10.1016/j.bmc.2008.09.071.

20. J. Coro, R. Atherton, S. Little, H. Wharton, V. Yardley, A. Alvarez Jr., M. Suarez, R. Perez and H. Rodriguez, Bioorg. Med. Chem., 16, 1312 (2006); https://doi.org/10.1016/j.bmcl.2005.11.060.

21. V. Marakov, O.B. Riabova, A. Yuschenko, N. Urlyapova, A. Daudova, P.F. Ziplef and U. Mollmann, J. Antimicrob. Chemother, 57, 1134 (2006); https://doi.org/10.1093/jac/dk1095.

22. S.L. Cao, Y.P. Feng, X.L. Zheng, Y.Y. Jiang, M. Zhang, Y. Wang and M. Xu, Arch. Pharm. Chem. Life Sci., 339, 250 (2006); https://doi.org/10.1002/ardp.200500264.

23. X. Hou, Z. Ge, T. Wang, W. Guo, J. Wu, J. Cui, C. Lai and R. Li, Arch. Pharm. Chem. Life Sci., 344, 320 (2011); https://doi.org/10.1002/ardp.201000259.

24. A. Goel, S.J. Mazur, R.J. Fattah, T.L. Hartman, J.A. Turpin, M. Huang, W.G. Rice, E. Appella and J.K. Inman, Bioorg. Med. Chem. Lett., 12, 767 (2002) https://doi.org/10.1016/S0960-894X(02)00007-0.

25. X.Y. He, L. Lu, J. Qiu, P. Zou, F. Yu, X.K. Jiang, L. Li, S. Jiang, S. Liu and L. Xie, Bioorg. Med. Chem., 21, 7539 (2013); https://doi.org/10.1016/j.bmc.2013.04.046.

26. S.L. Cao, Y. Han, C.Z. Yuan, Y. Wang, Z.K. Xiahou, J. Liao, R.T. Gao, B.B. Mao, B.L. Zhao, Z.F. Li and X. Xu, Eur. J. Med. Chem., 64, 401 (2013); https://doi.org/10.1016/j.eimech.2013.04.017.

27. S. Jangir, V. Bala, N. Lal, L. Kumar, A. Sarswat, A. Kumar, Hamidullah, K.S. Saini, V. Sharma, V. Verma, J.P. Maikhuri, R. Konwar, G. Gupta and V.L. Sharma, Eur. J. Med. Chem., 85, 638 (2014); https://doi.org/10.1016/j.ejmech.2014.08.028.

28. P. Liu, C. Li, J. Zhang and X. Xu, Synth. Commun., 43, 3342 (2013); https://doi.org/10.1080/00397911.2013.783600.
29. A.Z. Halimehjani, Y. Pourshojaei and M.R. Saidi, Tetrahedron Lett., 50, 32 (2009); https://doi.org/10.1016/j.tetlet.2008.10.063.

30. J. Jamir, U.B. Sinha, J. Nath and B.K. Patel, Synth. Commun., 42, 951 (2012); https://doi.org/10.1080/00397911.2010.532276.

31. M. Kienle, A. Unsinn and P. Knochel, Angew. Chem. Int. Ed., 49, 4751 (2010); https://doi.org/10.1002/anie.201001025.

32. V. Aucagne, C. Lorin, A. Tatibouet and P. Rollin, Tetrahedron Lett., 46, 4349 (2005); https://doi.org/10.1016/j.tetlet.2005.04.112.

33. K. Alagiri and K.R. Prabhu, Chem. Eur. J., 17, 6922 (2011); https://doi.org/10.1002/chem.201100817.

34. N.K. Kumar, K. Sreeramamurthy, S. Palle, K. Mukkanti and P. Das, Tetrahedron Lett., 51, 899 (2010); https://doi.org/10.1016/j.tetlet.2009.11.127.

35. M.A. Khalilzadeh, Z. Hossaini, M.M. Baradarani and A. Hasannia, Tetrahedron Lett., 66, 8464 (2010); https://doi.org/10.1016/i.tet.2010.08.041.

36. J.Y. Park, I.A. Ryu, H.H. Park, D.C. Ha and Y.D. Gong, Synthesis, 913 (2009); https://doi.org/10.1055/s-0028-1087970.

37. D. Katiyar, V.K. Tiwari, R.P. Tripathi, A. Srivastava, V. Chaturvedi, R. Srivastava and B.S. Srivastava, Bioorg. Med. Chem., 11, 4369 (2003); https://doi.org/10.1016/S0968-0896(03)00480-2.

38. P. Das, C.K. Kumar, N. Kumar, M. Innus, J. Iqbal and N. Srinivas, Tetrahedron Lett., 49, 992 (2008);

39. U.A. Mohsen, Cukurova Med. J., 39, 729 (2014); https://doi.org/10.17826/cutf.79473.

40. S.K. Tandel, S. Rajappa and S.V. Pansare, Tetrahedron, 49, 7479 (1993); https://doi.org/10.1016/S0040-4020(01)87224-0.

41. F.R. Charati, Z. Hossaini and R. Hajinasiri, J. Appl. Chem. Res., 20, 54 (2012)

42. L.J. Bahrin, P.J. Jones and H. Hopf, Beilstein J. Org. Chem., 8, 1999 (2012); https://doi.org/10.3762/bjoc.8.226.

43. N.S. Gu“nay, G. Capan, N. Ulusoy, N. Ergenc, G. Ötük and D. Kaya, Il Farmaco, 54, 826 (1999); https://doi.org/10.1016/S0014-827X(99)00109-3.

44. K. Chauhan, M. Sharma, P. Singh, V. Kumar, P.K. Shukla, M.I. Siddiqi and P.M.S. Chauhan, MedChemComm, 3, 1104 (2012); https://doi.org/10.1039/c2md20109g.

45. P.M. Madalageri and O. Kotresh, Der Pharm. Chem., 4, 2187 (2012).

46. C. Donnici, L. Nogueira, M. Araujo, S. Oliveira, T. Magalhães, M. Lopes, A. Silva, A. Ferreira, C. Martins and M. de Resende Stoianoff, Molecules, 19, 5402 (2014); https://doi.org/10.3390/molecules 19045402.

47. E. Septolu, M.D. Aytemr, E. Killic, M. Ozalp and U. Califi, FABAD J. Pharm. Sci., 29, 1 (2004)

48. D. Chaturvedi, S. Ray, A.K. Srivastava and R. Chander, Bioorg. Med. Chem., 16, 2489 (2008); https://doi.org/10.1016/i.bmc.2007.11.062. 